\title{
Approximation Limits of Linear Programs (Beyond Hierarchies)
}

\author{
Gábor Braun ${ }^{1}$, Samuel Fiorini' ${ }^{2}$, Sebastian Pokutta ${ }^{3}$, and David Steurer ${ }^{4}$ \\ ${ }^{1}$ Universität Leipzig, Institut für Informatik, PF 100920, 04009 Leipzig, Germany. \\ Email: gabor.braun@informatik.uni-leipzig.de \\ ${ }^{2}$ Department of Mathematics, Université libre de Bruxelles CP 216, Bd. du Triomphe, 1050 Brussels, \\ Belgium. Email: sfiorini@ulb.ac.be \\ ${ }^{3}$ ISyE, Georgia Institute of Technology, Atlanta, GA, USA. Email: sebastian.pokutta@isye.gatech.edu \\ ${ }^{4}$ Department of Computer Science, Cornell University, Ithaca, NY 14853, United States. \\ Email: dsteurer@cs.princeton.edu
}

May 20, 2014

\begin{abstract}
We develop a framework for proving approximation limits of polynomial-size linear programs from lower bounds on the nonnegative ranks of suitably defined matrices. This framework yields unconditional impossibility results that are applicable to any linear program as opposed to only programs generated by hierarchies. Using our framework, we prove that $O\left(n^{1 / 2-\epsilon}\right)$-approximations for CLIQUE require linear programs of size $2^{n^{\Omega(\epsilon)}}$. This lower bound applies to linear programs using a certain encoding of CLIQUE as a linear optimization problem. Moreover, we establish a similar result for approximations of semidefinite programs by linear programs.

Our main technical ingredient is a quantitative improvement of Razborov's rectangle corruption lemma (1992) for the high error regime, which gives strong lower bounds on the nonnegative rank of shifts of the unique disjointness matrix.
\end{abstract}

\section{Introduction}

Linear programs (LPs) play a central role in the design of approximation algorithms, see, e.g., [Vazirani, 2001, Williamson and Shmovs, 2011, Lau et al., 2011]. Therefore, understanding the limitations of LPs as tools for designing approximation algorithms is an important question.

The first generation of results studied the limitations of specific LPs by seeking to determine their integrality gaps. The second generation of results, pioneered by Arora et al. [2002], studied the limitations of structured LPs such as those generated by lift-and-project procedures or hierarchies (e.g., Sherali and Adams [1990] and Lovász and Schrijver [1991]).

In this work, we start a third generation of results that apply to any LP for a given problem. For example, our lower bounds address the following question: Is there a polynomial-size linear programming relaxation $\mathrm{LP}_{n}$ for CLIQUE that achieves a $n^{\Theta(1)}$-approximation for all graphs with at most $n$ vertices? We develop a framework for reducing questions of this kind to lower bounds on the nonnegative rank 1 of certain matrices associated to the problem, and then prove lower bounds for the matrices corresponding to CLIQUE.

\footnotetext{
${ }^{1}$ The nonnegative rank of a matrix $M$, denoted $\operatorname{rank}_{+}(M)$, is the minimum $r$ such that $M=T U$ where $T$ and $U$ are nonnegative matrices with $r$ columns and $r$ rows, respectively.
} 
The matrices studied here are related to the unique disjointness problem, a variant of the famous disjointness problem from communication complexity (see, e.g., Chattopadhyay and Pitassi [2010] for a survey). In the disjointness problem (DISJ), both Alice and Bob receive a subset of $[n]:=$ $\{1, \ldots, n\}$. They have to determine whether the two subsets are disjoint. The unique disjointness problem (UDISJ) is the promise version of the disjointness problem where the two subsets are guaranteed to have at most one element in common. Denoting the binary encoding of the sets of Alice and Bob by $a, b \in\{0,1\}^{n}$, respectively, this amounts to computing the Boolean function $\operatorname{UDISJ}(a, b):=1-a^{\top} b$ on the set of pairs $(a, b) \in\{0,1\}^{n} \times\{0,1\}^{n}$ with $a^{\top} b \in\{0,1\}$. Viewing it as a partial $2^{n} \times 2^{n}$ matrix, we call UDISJ the unique disjointness matrix.

It is known that the communication complexity of UDISJ is $\Omega(n)$ bits for deterministic, nondeterministic and even randomized communication protocols Kalyanasundaram and Schnitger, 1992, Razborov, 1992, Bar-Yossef et al., 2004]. One consequence of this is that the nonnegative rank of any matrix obtained from UDISJ by filling arbitrarily the blank entries (for pairs $(a, b)$ with $a^{\top} b>1$ ) and perhaps adding rows and/or columns is still $2^{\Omega(n)}$. Indeed: (i) the support of the resulting matrix has $\Omega(n)$ nondeterministic communication complexity because it contains UDISJ, (ii) for every matrix $M, \log \operatorname{rank}_{+}(M)$ is lower bounded by the nondeterministic communication complexity of (the support matrix of) $M$ [Yannakakis, 1991].

In a recent paper Fiorini et al. [2012] proved strong lower bounds on the size of LPs expressing the traveling salesman problem (TSP), or more precisely on the size of extended formulations of the TSP polytope (see Section_ for definitions of concepts related to polyhedra, extended formulations and slack matrices). Their proof works by embedding UDISJ in a slack matrix of the TSP polytope of the complete graph on $\Theta\left(n^{2}\right)$ vertices. This solved a question left open in Yannakakis [1991]. We use a similar approach for approximate extended formulations. In case of CLIQUE, our approach requires lower bounds on the nonnegative rank of partial matrices obtained from the UDISJ matrix by adding a positive offset to all the entries.

\subsection{Related Work}

Our results are closely related to previous work in communication complexity for the (unique) disjointness problem and related problems. Lower bounds of $\Omega(n)$ on the randomized, bounded error communication complexity of disjointness were established in Kalyanasundaram and Schnitger [1992]. In Razborov [1992] the distributional complexity of unique disjointness problem was analyzed, which in particular implies the result of Kalyanasundaram and Schnitger [1992]. In that famous paper, Razborov proved the following rectangle corruption lemma: for every large rectangle within UDISJ, the number of 0 -entries is proportional to the number of 1-entries.

The most recent proof that the randomized, bounded error communication complexity of DISJ is $\Omega(n)$ is due to Bar-Yossef et al. [2004] and is based on information theoretic arguments. This leads to a lower bound for randomized communication within a high-error regime, that is, when the error probability is close to $1 / 2$. Here we derive a strong generalization dealing with shifts for approximate EFs and we recover the high-error regime bound.

There has been extensive work on LP and SDP hierarchies/relaxations and their limitations; we will be only able to list a few here. In Charikar et al. [2009], strong lower bounds (of $2-\epsilon$ ) on the integrality gap for $n^{\epsilon}$ rounds of the Sherali-Adams hierarchy when applied to (natural relaxations of) VERTEX COVER, Max CUT, SPARSEST CUT have been been established via embeddings into $\ell_{2}$; see also Charikar et al. [2010] for limits and tradeoffs in metric embeddings. For integrality gaps of relaxations for the KNAPSACK problem see Karlin et al. [2011]. A nice overview of the differences and similarities of the Sherali-Adams, the Lovász-Schrijver and the Lasserre hierarchies/relaxations can be found in Laurent [2003].

Similar to the level of a hierarchy, we have the notion of rank for the Lovász-Schrijver relax- 
ation and rank correspond to a similar complexity measure as the level. The rank is the minimum number of application of the Lovász-Schrijver operator $N$ until we obtain the integral hull of the polytope under consideration. Rank lower bounds of $n$ for Lovász-Schrijver relaxations of CLIQUE have been obtained in Cook and Dash [2001]; a similar result for Sherali-Adams hierarchy can be found in Laurent [2003].

In Singh and Talwar [2010] integrality gaps, after adding few rounds of Chvátal-Gomory cuts, have been studied for problems including $k$-CSP, Max CUT, VERTEX COVER, and UNIQUE LABEL COVER showing that in some cases (e.g., $k$-CSP) the gap can be significantly reduced whereas in most other cases the gap remains high.

In the context of SDP relaxations, in particular formulations derived from the Lovász-Schrijver $N_{+}$hierarchies (see Lovász and Schrijver [1991]) and the Lasserre hierarchies (see Lasserre [2002]) there has been significant work in recent years. For example, Arora et al. [2009] obtained a $O(\sqrt{\log n})$ upper bound on a suitable SDP relaxation of SPARSEST CUT. For lower bounds in terms of rank, see e.g., Schoenebeck [2008] for the $k$-CSP in the Lasserre hierarchy or Schoenebeck et al. [2007] for VERTEX COVER in the semidefinite Lovász-Schrijver hierarchy. Motivated by the Unique Games Conjecture, several works studied upper and lower bounds for SDP hierarchy relaxations of Unique Games (see for example, Guruswami and Sinop [2011], Barak et al. [2011, 2012b,a]).

Approximate extended formulations have been studied before, for specific problems, e.g., KNAPSACK in Bienstock [2008], or as a general tool, see Vyve and Wolsev [2006].

For recent results on computing the nonnegative rank see, e.g., Arora et al. [2012].

\subsection{Contribution}

The contribution of the present paper is threefold.

(i) We develop a framework for proving lower bounds on the sizes of approximate EFs. Through a generalization of Yannakakis's factorization theorem, we characterize the minimum size of a $\rho$-approximate extended formulations as the nonnegative rank of any slack matrix of a pair of nested polyhedra. Thus we reduce the task of proving approximation limits for LPs to the task of obtaining lower bounds on the nonnegative ranks of associated matrices. Typically, these matrices have no zeros, which renders it impossible to use nondeterministic communication complexity. We emphasize the fact that the results obtained within our framework are unconditional. In particular, they do not rely on $\mathrm{P} \neq \mathrm{NP}$.

(ii) We extend Razborov's rectangle corruption lemma to deal with shifts of the UDISJ matrix. As a consequence, we prove that the nonnegative rank of any matrix obtained from the UDISJ matrix by adding a constant offset to every entry is still $2^{\Omega(n)}$. Moreover, we show that the nonnegative rank is still $2^{\Omega\left(n^{2 \epsilon}\right)}$ when the offset is at most $n^{1 / 2-\epsilon}$. To our knowledge, these are the first strong lower bounds on the nonnegative rank of matrices that contain no zeros. Our extension of Razborov's lemma allow us to recover known lower bounds for DISJ in the high-error regime of Bar-Yossef et al. [2004].

(iii) We obtain a strong hardness result for CLIQUE w.r.t. a natural linear encoding of the problem. From the results described above, we prove that the size of every $O\left(n^{1 / 2-\epsilon}\right)$-approximate EF for CLIQUE is $2^{\Omega\left(n^{2 \epsilon}\right)}$. Finally, we observe that the same bounds hold for approximations of SDPs by LPs. This suggests that SDP-based approximation algorithms can be significantly stronger than LP-based approximation algorithms. The inapproximability of SDPs by LPs has some interesting consequences. In particular we cannot expect to convert SDP-based approximation algorithms into LP-based ones by approximating the PSD-cone via linear programming. 
We point out that our framework readily generalizes to SDPs by replacing nonnegative rank with PSD rank (see Gouveia et al. [2013a] for a definition of the PSD rank). However, no strong bound on PSD rank seems to be currently in sight.

Finally, we report that the results of this paper have inspired further research.

- Braverman and Moitra [2013] improved our lower bound on the nonnegative rank of shifted UDISJ matrices and obtain super-polynomial lower bounds for shifts up to $O\left(n^{1-\epsilon}\right)$, hence matching the algorithmic hardness of approximation for CLIQUE. This was achieved by pioneering information-theoretic methods for proving lower bounds on the nonnegative rank. An alternative information theoretic approach for lower bounding the nonnegative rank which simplifies and slightly improves the results in Braverman and Moitra [2013] has been presented in Braun and Pokutta [2013]. This last paper also establishes that matrices obtained from shifts of UDISJ by removing rows and columns, or flipping entries, still have high nonnegative rank.

- Chan et al. [2013] obtain lower bounds on the size of LPs approximating Max CSP. In particular, they prove that approximating Max CUT (with nonnegative weights) with a constant factor less than 2 requires $n^{\Omega(\log n / \log \log n)}$. This solves a conjecture we stated in an earlier version of this text.

- Rothvoß [2014] proved a $2^{\Omega(n)}$ lower bound on the nonnegative rank of the slack matrix of the perfect matching polytope by a significant modification of Razborov's lemma. This exciting result essentially proves that there are is no small LP that can solve all weighted instance of the matching problem on a $n$-vertex complete graph.

\subsection{Outline}

We begin in Section 2 by setting up our framework for studying approximate extended formulations of combinatorial optimization problems. Then we extend Razborov's rectangle corruption lemma in Section 3 and use this to prove strong lower bounds on the nonnegative rank of shifts of the UDISJ matrix. Finally, we draw consequences for CLIQUE and approximations of SDPs by LPs in Section 4 .

\section{Framework for Approximation Limits of LPs}

In this section we establish our framework for studying approximation limits of LPs. First, we define in details the concepts of linear encodings and approximate extended formulations. Second, we prove a factorization theorem for pairs of nested polyhedra reducing existential questions on approximate extended formulations to the computation of nonnegative ranks of corresponding slack matrices.

\subsection{Preliminaries}

A (convex) polyhedron is a set $P \subseteq \mathbb{R}^{d}$ that is the intersection of a finite collection of closed halfspaces. In other words, $P$ is a polyhedron if and only if $P$ is the set of solutions of a finite system of linear inequalities and possibly equalities. (Note that every equality can be represented by a pair of inequalities.) Equivalently, a set $P \subseteq \mathbb{R}^{d}$ is a polyhedron if and only if $P$ is the Minkowski sum of the convex hull conv $(V)$ of a finite set $V$ of points and the conical hull cone $(R)$ of a finite set $R$ of vectors, that is, $P=\operatorname{conv}(V)+$ cone $(R)$.

Let $P \subseteq \mathbb{R}^{d}$ be a polyhedron. The dimension of $P$ is the dimension of its affine hull aff $(P)$. A face of $P$ is a subset $F:=\left\{x \in P \mid w^{\top} x=\delta\right\}$ such that $P$ satisfies the inequality $w^{\top} x \leqslant \delta$. Note that face $F$ is again a polyhedron. A vertex is a face of dimension 0 , i.e., a point. A facet is a face of 
dimension one less than $P$. The inequality $w^{\top} x \leqslant \delta$ is called facet-defining if the face $F$ it defines is a facet. The recession cone rec $(P)$ of $P$ is the set of directions $v \in \mathbb{R}^{d}$ such that, for a point $p$ in $P$, all points $p+\lambda v$ where $\lambda \geqslant 0$ belong to $P$. The recession cone of $P$ does not depend on the base point $p$, and is again a polyhedron (even more, it is a polyhedral cone). The elements of the recession cone are sometimes called rays.

A (convex) polytope $P \subseteq \mathbb{R}^{d}$ is a bounded polyhedron. Equivalently, $P$ is a polytope if and only if $P$ is the convex hull conv $(V)$ of a finite set $V$ of points. Let $P \subseteq \mathbb{R}^{d}$ be a polytope. Every (finite or infinite) set $V$ such that $P=\operatorname{conv}(V)$ contains all the vertices of $P$. Letting vert $(P)$ denote the vertex set of $P$, then we have $P=\operatorname{conv}(\operatorname{vert}(P))$. Every (finite) system describing $P$ contains all the facet-defining inequalities of $P$, up to scaling by positive numbers and adding equalities satisfied by all points of $P$. Conversely, a linear description of $P$ can be obtained by picking one defining inequality per facet and adding a system of equalities describing $\operatorname{aff}(P)$. A 0/1-polytope in $\mathbb{R}^{d}$ is simply the convex hull of a subset of $\{0,1\}^{d}$.

For more about convex polytopes and polyhedra, see the standard reference Ziegler [1995].

\subsection{Linear Encodings of Problems and Approximate EFs}

A linear encoding of a (combinatorial optimization) problem is a pair $(\mathcal{L}, \mathcal{O})$ where $\mathcal{L} \subseteq\{0,1\}^{*}$ is the set of feasible solutions to the problem and $\mathcal{O} \subseteq \mathbb{R}^{*}$ is the set of admissible objective functions. An instance of the linear encoding is a pair $(d, w)$ where $d$ is a positive integer and $w \in \mathcal{O} \cap \mathbb{R}^{d}$. Solving the instance $(d, w)$ means finding $x \in \mathcal{L} \cap\{0,1\}^{d}$ such that $w^{\top} x$ is either maximum or minimum, according to the type of problem under consideration.

Example 1 (Linear encoding of metric TSP). In the natural linear encoding of the metric traveling salesman problem (metric TSP), the feasible solutions $x \in \mathcal{L}$ are the characteristic vectors (or incidence vectors) of tours of the complete graph over $[n]$ for some $n \geqslant 3$, and the admissible objective functions $w \in \mathcal{O}$ are all nonnegative vectors $w=\left(w_{i j}\right)$ such that $w_{i k} \leqslant w_{i j}+w_{j k}$ for all distinct $i$, $j$ and $k$ in $[n]$. All vectors are encoded in $\mathbb{R}^{d}$, where $d=\left(\begin{array}{l}n \\ 2\end{array}\right)$. By considering all possible $n \geqslant 3$, we obtain the pair $(\mathcal{L}, \mathcal{O})$ corresponding to metric TSP. (Recall that metric TSP is a minimization problem.)

For every fixed dimension $d$, a linear encoding $(\mathcal{L}, \mathcal{O})$ naturally defines a pair of nested convex sets $P \subseteq Q$ where

$$
\begin{aligned}
& P:=\operatorname{conv}\left(\left\{x \in\{0,1\}^{d} \mid x \in \mathcal{L}\right\}\right), \text { and } \\
& Q:=\left\{x \in \mathbb{R}^{d} \mid \forall w \in \mathcal{O} \cap \mathbb{R}^{d}: w^{\top} x \leqslant \max \left\{w^{\top} z \mid z \in P\right\}\right\}
\end{aligned}
$$

if the goal is to maximize and $Q:=\left\{x \in \mathbb{R}^{d} \mid \forall w \in \mathcal{O} \cap \mathbb{R}^{d}: w^{\top} x \geqslant \min \left\{w^{\top} z \mid z \in P\right\}\right\}$ if the goal is to minimize. Intuitively, the vertices of $P$ encode the feasible solutions of the problem under consideration and the defining inequalities of $Q$ encode the admissible objective functions. Notice that $P$ is always a $0 / 1$-polytope but $Q$ might be unbounded and, in some pathological cases, nonpolyhedral. Below, we will mostly consider the case where $Q$ is polyhedral, that is, defined by a finite number of "interesting" inequalities.

Given a linear encoding $(\mathcal{L}, \mathcal{O})$ of a maximization problem, and $\rho \geqslant 1$, a $\rho$-approximate extended formulation (EF) is an extended formulation $E x+F y=g, y \geqslant 0$ with $(x, y) \in \mathbb{R}^{d+r}$ such that

$$
\begin{aligned}
& \max \left\{w^{\top} x \mid E x+F y=g, y \geqslant 0\right\} \geqslant \max \left\{w^{\top} x \mid x \in P\right\} \quad \text { for all } \quad w \in \mathbb{R}^{d} \text { and } \\
& \max \left\{w^{\top} x \mid E x+F y=g, y \geqslant \mathbf{0}\right\} \leqslant \rho \max \left\{w^{\top} x \mid x \in P\right\} \quad \text { for all } w \in \mathcal{O} \cap \mathbb{R}^{d} .
\end{aligned}
$$


Letting $K:=\left\{x \in \mathbb{R}^{d} \mid \exists y \in \mathbb{R}^{r}: E x+F y=g, y \geqslant 0\right\}$, we see that this is equivalent to $P \subseteq K \subseteq \rho Q$. For a minimization problem, we require

$$
\begin{aligned}
& \min \left\{w^{\top} x \mid E x+F y=g, y \geqslant \mathbf{0}\right\} \leqslant \min \left\{w^{\top} x \mid x \in P\right\} \quad \text { for all } \quad w \in \mathbb{R}^{d} \text { and } \\
& \min \left\{w^{\top} x \mid E x+F y=g, y \geqslant \mathbf{0}\right\} \geqslant \rho^{-1} \min \left\{w^{\top} x \mid x \in P\right\} \quad \text { for all } w \in \mathcal{O} \cap \mathbb{R}^{d} .
\end{aligned}
$$

This is equivalent to $P \subseteq K \subseteq \rho^{-1} Q$.

Example 2 (Approximate extended formulation of metric TSP). We return to Example 1. It is known that the Held-Karp relaxation $K$ of the metric TSP has integrality gap at most 3/2 (see Held and Karp [1970], Wolsey [1980]). In geometric terms, this means that $P \subseteq K \subseteq 2 / 3 \cdot Q$. Although $K$ is defined by an exponential number of inequalities, it is known that it can be reformulated with a polynomial number of constraints by adding a polynomial number of variables, see, e.g., Carr et al. [2009]. That is, the Held-Karp relaxation $K$ has a polynomial-size extended formulation. Thus, the pair $(\mathcal{L}, \mathcal{O})$ for the metric TSP has a polynomial-size $3 / 2$-approximate EF.

We require the following faithfulness condition: every instance of the problem can be mapped to an instance of the linear encoding in such a way that feasible solutions to an instance of the problem can be converted in polynomial time to feasible solutions to the corresponding instance of the linear encoding without deteriorating their objective function values, and vice-versa. Roughly speaking, we ask that each instance of the problem can be encoded as an instance of the linear encoding.

For linear encoding of graph problems, such as the maximum clique problem (CLIQUE), the set of feasible solutions is not allowed to depend on the input graph, which therefore must be encoded solely in the objective function. The set of feasible solutions is only allowed to depend on the size $n$ of the ground set.

Example 3 (Max $k$-SAT). Consider the maximum $k$-SAT problem (Max $k$-SAT), where $k$ is constant. Letting $u_{1}, \ldots, u_{n}$ denote the variables of a Max $k$-SAT instance, we encode the problem in dimension $d=\Theta\left(n^{k}\right)$. For each nonempty clause $C$ of size at most $k$, we introduce a variable $x_{C}$. Collectively, these variables define a point $x \in \mathbb{R}^{d}$. Given a truth assignment, we set $x_{C}$ to 1 if $C$ is satisfied and otherwise we set $x_{C}$ to 0 . Letting $n$ vary, this defines a language $\mathcal{L} \subseteq\{0,1\}^{*}$. We let $\mathcal{O}:=\{0,1\}^{*}$.

The pair $(\mathcal{L}, \mathcal{O})$ defines a linear encoding of Max $k$-SAT because each instance of Max $k$-SAT can be encoded as an instance of $(\mathcal{L}, \mathcal{O})$. More precisely, to any given set of clauses over $n$ variables, we can associate a dimension $d=\Theta\left(n^{k}\right)$ and weight vector $w \in\{0,1\}^{d}$ such that maximizing $\sum w_{C} x_{C}$ for $x \in \mathcal{L} \cap\{0,1\}^{d}$ corresponds to finding a truth assignment that maximizes the number of satisfied clauses.

Finally, we remark that the EF defined by the inequalities $0 \leqslant x_{C} \leqslant 1$ and $x_{C} \leqslant \sum_{u_{i} \in C} x_{\left\{u_{i}\right\}}+$ $\sum_{\bar{u}_{i} \in C}\left(1-x_{\left\{u_{i}\right\}}\right)$ for all clauses $C$ is a polynomial-size 4/3-approximate EF for Max $k$-SAT, as follows from Goemans and Williamson [1994].

\subsection{Factorization Theorem for Pairs of Nested Polyhedra}

Let $P$ and $Q$ be polyhedra with $P \subseteq Q \subseteq \mathbb{R}^{d}$. An extended formulation (EF) of the pair $P, Q$ is a system $E x+F y=g, y \geqslant \mathbf{0}$ defining a polyhedron $K:=\left\{x \in \mathbb{R}^{d} \mid E x+F y=g, y \geqslant \mathbf{0}\right\}$ such that $P \subseteq K \subseteq Q$. We denote by $\mathrm{xc}(P, Q)$ the minimum size of an EF of the pair $P, Q$.

Now consider an inner description $P:=\operatorname{conv}\left(\left\{v_{1}, \ldots, v_{n}\right\}\right)+\operatorname{cone}\left(\left\{r_{1}, \ldots, r_{k}\right\}\right)$ of $P$ and an outer description $Q:=\left\{x \in \mathbb{R}^{d} \mid A x \leqslant b\right\}$ of $Q$, where the system $A x \leqslant b$ consists of $m$ inequalities: $A_{1} x \leqslant b_{1}, \ldots, A_{m} x \leqslant b_{m}$. The slack matrix of the pair $P, Q$ w.r.t. these inner and outer descriptions is 
the $m \times(n+k)$ matrix $S^{P, Q}=\left[S_{\text {vertex }}^{P, Q} S_{\text {ray }}^{P, Q}\right]$ given by block decomposition into a vertex and ray part:

$$
\begin{aligned}
S_{\text {vertex }}^{P, Q}(i, j) & :=b_{i}-A_{i} v_{j}, & & i \in[m], j \in[n], \\
S_{\text {ray }}^{P, Q}(i, j) & :=-A_{i} r_{j}, & & i \in[m], j \in[k] .
\end{aligned}
$$

A rank-r nonnegative factorization of an $m \times n$ matrix $M$ is a decomposition of $M$ as a product $M=T U$ of nonnegative matrices $T$ and $U$ of sizes $m \times r$ and $r \times n$, respectively. The nonnegative rank $\operatorname{rank}_{+}(M)$ of $M$ is the minimum rank $r$ of nonnegative factorizations of $M$. In case $M$ is zero, we let $\operatorname{rank}_{+}(M)=0$. It is quite useful to notice that the nonnegative rank of $M$ is also the minimum number of nonnegative rank-1 matrices whose sum is $M$. From this, we see immediately that the nonnegative rank of $M$ is at least the nonnegative rank of any of its submatrices.

Our first result gives an essentially exact characterization of $\operatorname{xc}(P, Q)$ in terms of the nonnegative rank of the slack matrix of the pair $P, Q$. It states that the minimum extension complexity $\mathrm{xc}(P, Q)$ of a polyhedron sandwiched between $P$ and $Q$ equals the nonnegative rank of $S^{P, Q}$ (minus 1 , in some cases). The result readily generalizes Yannakakis's factorization theorem [Yannakakis, 1991], which concerns the case $P=Q$. The idea of considering a pair $P, Q$ as we do here first appeared in Pashkovich [2012] and similar ideas appeared earlier in Gillis and Glineur [2012].

Theorem 1. With the above notations, we have $\operatorname{rank}_{+}\left(S^{P, Q}\right)-1 \leqslant \mathrm{xc}(P, Q) \leqslant \operatorname{rank}_{+}\left(S^{P, Q}\right)$ for every slack matrix of the pair $P, Q$. If the affine hull of $P$ is not contained in $Q$ and $\operatorname{rec}(Q)$ is not full-dimensional, we have $\mathrm{xc}(P, Q)=\operatorname{rank}_{+}\left(S^{P, Q}\right)$. In particular, this holds when $P$ and $Q$ are polytopes of dimension at least 1.

Proof. First, we deal with degenerate cases. Observe that $\operatorname{xc}(P, Q)=0$ if and only if there exists an affine subspace containing $P$ and contained in $Q$, that is, if and only if the affine hull of $P$ is contained in $Q$. In this case, we have $\operatorname{rank}_{+}\left(S^{P, Q}\right) \in\{0,1\}$, so the theorem holds.

Now assume that the affine hull of $P$ is not contained in $Q$. Then, $\operatorname{rank}_{+}\left(S^{P, Q}\right) \geqslant 1$ because having $\operatorname{rank}_{+}\left(S^{P, Q}\right)=0$ means either that $S^{P, Q}$ is empty, that is, $m=0$ or $n+k=0$, or that $S^{P, Q}$ is the zero matrix. In all cases, this contradicts our assumption that the affine hull of $P$ is not contained in $Q$.

Next, let $S^{P, Q}=T U$ be any rank-r nonnegative factorization of $S^{P, Q}$ with $r=\operatorname{rank}_{+}\left(S^{P, Q}\right) \geqslant 1$. This factorization decomposes into blocks: $S_{\text {vertex }}^{P, Q}=T U_{\text {vertex }}$ and $S_{\text {ray }}^{P, Q}=T U_{\text {ray }}$. Consider the system

$$
A x+T y=b, y \geqslant 0
$$

and the corresponding polyhedron $K:=\left\{x \in \mathbb{R}^{d} \mid A x+T y=b, y \geqslant \mathbf{0}\right\}$.

We verify now that $P \subseteq K \subseteq Q$. The inclusion $K \subseteq Q$ simply follows from $T y \geqslant 0$. For the inclusion $P \subseteq K$, pick a vertex $v_{j}$ of $P$ and observe that $(x, y)=\left(v_{j}, U_{\text {vertex }}^{j}\right)$ satisfies (1), where $U_{\text {vertex }}^{j}$ denotes the $j$ th column of $U_{\text {vertex }}$, because $A v_{j}+T U_{\text {vertex }}^{j}=A v_{j}+b-A v_{j}=b$ and $U^{j} \geqslant \mathbf{0}$. Similarly, for every ray $r_{j}$ we obtain a ray $\left(r_{j}, U_{\text {ray }}^{j}\right)$ of $K$ as $A r_{j}+T U_{\text {ray }}^{j}=0$ and $U_{\text {ray }}^{j} \geqslant \mathbf{0}$.

Thus we obtain that (1) is a size-r EF of the pair $P, Q$. Therefore, $\mathrm{xc}(P, Q) \leqslant \operatorname{rank}_{+}\left(S^{P, Q}\right)$.

Finally, suppose that the system

$$
E x+F y=g, y \geqslant 0
$$

defines a size- $r$ EF of the pair $P, Q$. Let $L \subseteq \mathbb{R}^{d+r}$ denote the polyhedron defined by (2), and let $K \subseteq \mathbb{R}^{d}$ denote the orthogonal projection of $L$ into $x$-space.

Since $P \subseteq K$, for each point $v_{j}$, there exists $w_{j} \in \mathbb{R}_{+}^{r}$ such that $\left(v_{j}, w_{j}\right) \in L$. Similarly, for each ray $r_{j}$ there exists a $z_{j} \in \mathbb{R}_{+}^{r}$ with $\left(r_{j}, z_{j}\right)$ a ray of $L$. Let $W$ be the matrix with columns $w_{j}$, and $Z$ be the matrix with columns $z_{j}$. 
Since $K \subseteq Q$, by Farkas's lemma, $A x \leqslant b$ can be derived from (2), i.e., there exists a matrix $T$ and a vector $c \geqslant 0$ with $A=T E, b=T g+c$ and $T F \geqslant 0$. This gives the factorizations $S_{\text {vertex }}^{P, Q}=$ $(T F) W+c \mathbf{1}^{\top}$ and $S_{\text {ray }}^{P, Q}=(T F) Z$, resulting in the rank- $(r+1)$ nonnegative factorization $S^{P, Q}=$ $\left[\begin{array}{ll}T F & c\end{array}\right] \cdot\left[\begin{array}{cc}W & Z \\ \mathbf{1}^{\top} & \mathbf{0}^{\top}\end{array}\right]$. Taking $r=\mathrm{xc}(P, Q)$, we find $\operatorname{rank}_{+}\left(S^{P, Q}\right) \leqslant \mathrm{xc}(P, Q)+1$.

Finally, when $\operatorname{rec}(Q)$ is not full-dimensional, then $c$ above can be chosen to be $\mathbf{0}$. This simplifies the factorization, and yields the sharper inequality $\operatorname{rank}_{+}\left(S^{P, Q}\right) \leqslant \mathrm{xc}(P, Q)$.

Let $P, Q$ be as above and $\rho \geqslant 1$. Then $\rho Q=\left\{x \in \mathbb{R}^{d} \mid A x \leqslant \rho b\right\}$ and the slack matrix of the pair $P, \rho Q$ is related to the slack matrix of the pair $P, Q$ in the following way:

$$
\begin{gathered}
S_{\text {vertex }}^{P, \rho Q}(i, j)=\rho b_{i}-A_{i} v_{j}=(\rho-1) b_{i}+b_{i}-A_{i} v_{j}=S_{i j}^{P, Q}+(\rho-1) b_{i}, \\
S_{\text {ray }}^{P, \rho Q}(i, j)=S_{i j}^{P, Q} .
\end{gathered}
$$

Theorem 1 directly yields the following result.

Theorem 2. Consider a maximization problem with a linear encoding. Let $P, Q \subseteq \mathbb{R}^{d}$ be the pair of polyhedra associated with the linear encoding, and let $\rho \geqslant 1$. Consider any slack matrix $S^{P, Q}$ for the pair $P, Q$ and the corresponding slack matrix $S^{P, \rho Q}$ for the pair $P, \rho Q$. Then the minimum size of a $\rho$-approximate EF of the problem, w.r.t. the considered linear encoding, is $\operatorname{rank}_{+}\left(S^{P, \rho Q}\right)+\Theta(1)$, where the constant is 0 or 1. For a minimization problem, the minimum size of a $\rho$-approximate $E F$ is $\operatorname{rank}_{+}\left(S^{P, \rho^{-1} Q}\right)+\Theta(1)$.

Fixing $\rho \geqslant 1$, Theorem 2 characterizes the minimum number of inequalities in any LP providing a $\rho$-approximation for the problem under consideration. We point out that the theorem directly generalizes to SDPs, by replacing nonnegative rank by PSD rank [Gouveia et al., 2013a]. Here, we focus on LPs and nonnegative rank. As a matter of fact, strong lower bounds on the PSD rank seem to be currently lacking.

\subsection{A Problem with no Polynomial-Size Approximate EF}

We conclude this section with an example showing the necessity to restrict the set of admissible objective functions rather than allowing every $w \in \mathbb{R}^{*}$ (that is $P=Q$ ).

Let $K_{n}=\left(V_{n}, E_{n}\right)$ denote the $n$-vertex complete graph. For a set $X$ of vertices of $K_{n}$, we let $\delta(X)$ denote the set of edges of $K_{n}$ with one endpoint in $X$ and the other in its complement $\bar{X}$. This set $\delta(X)$ is known as the cut defined by $X$. For a subset $F$ of edges of $K_{n}$, we let $\chi^{F} \in \mathbb{R}^{E_{n}}$ denote the characteristic vector (or incidence vector) of $F$, with $\chi_{e}^{F}=1$ if $e \in F$ and $\chi_{e}^{F}=0$ otherwise. The cut polytope CUT $(n)$ is defined as the convex hull of the characteristic vectors of all cuts in the complete graph $K_{n}=\left(V_{n}, E_{n}\right)$. That is,

$$
\operatorname{CUT}(n):=\operatorname{conv}\left(\left\{\chi^{\delta(X)} \in \mathbb{R}^{E_{n}} \mid X \subseteq V_{n}\right\}\right) .
$$

A related object is the cut cone, defined as the cone generated by the cut-vectors $\chi^{\delta(X)}$ :

$$
\operatorname{CUT}-\operatorname{CONE}(n):=\text { cone }\left(\left\{\chi^{\delta(X)} \in \mathbb{R}^{E_{n}} \mid X \subseteq V_{n}\right\}\right) .
$$

Consider the maximum cut problem (Max CUT) with arbitrary weights, and its usual linear encoding. With this encoding we have $P=Q=\operatorname{CUT}(n)$. Our next result states that this problem has no $\rho$-approximate $\mathrm{EF}$, whatever $\rho \geqslant 1$ is. Intuitively, this phenomenon stems from the fact that, because $\mathbf{0}$ is a vertex of the cut polytope, every approximate EF necessarily 'captures' all facets of the cut polytope incident to $\mathbf{0}$ (see Figure 1). These facets define the cut cone, which turns out to have high extension complexity. Although this follows rather easily from ideas of Fiorini et al. [2012], we include a proof here for completeness. 

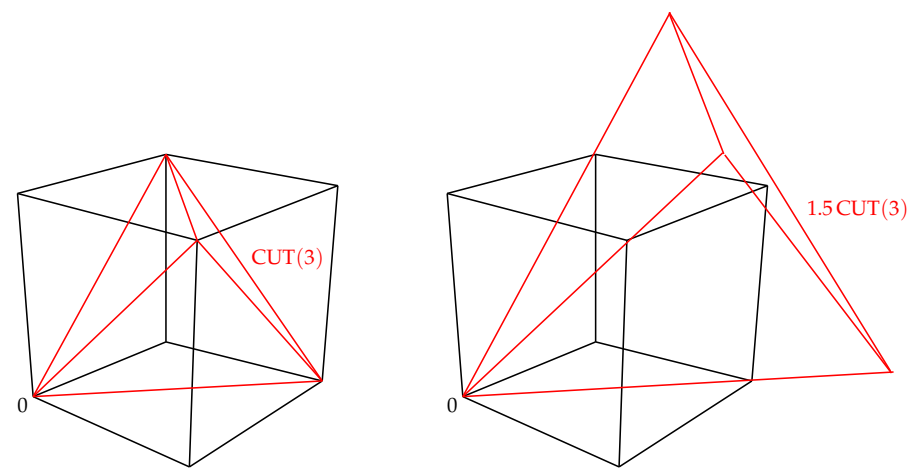

Figure 1: $\operatorname{CUT}(3)$ and a dilate $\rho \operatorname{CUT}(3)$ for $\rho=1.5$.

Proposition 3. For every $\rho \geqslant 1$, every $\rho$-approximate EF of the Max CUT problem with arbitrary weights has size $2^{\Omega(n)}$. More precisely, disregarding the value of $\rho \geqslant 1$, we have $\operatorname{xc}(\operatorname{CUT}(n), \rho \operatorname{CUT}(n))=2^{\Omega(n)}$.

Proof. Let $E x+F y=g, y \geqslant \mathbf{0}$ denote a minimum size $\rho$-approximate $\mathrm{EF}$ of $\mathrm{CUT}(n)$. We claim that

$$
E x+F y-\lambda g=0, y \geqslant 0, \lambda \geqslant 0
$$

is an EF of the cut cone. Let $K$ be the polyhedron obtained by projecting the set of solutions of (3) into $x$-space. Clearly, $K$ is a cone containing all the cut-vectors $\chi^{\delta(X)}$, from which we get that $\operatorname{CUT}-\operatorname{CONE}(n) \subseteq K$. Now take any point $(x, y, \lambda)$ satisfying (3). If $\lambda=0$ then necessarily $x=\mathbf{0}$ because $E x+F y=\mathbf{0}, y \geqslant \mathbf{0}$ defines the recession cone of a polyhedron that projects into $\rho \operatorname{CUT}(n)$, which is bounded. In this case we have $x=0 \in \operatorname{CUT}-\operatorname{CONE}(n)$. Assume that $\lambda>0$. Then $E \lambda^{-1} x+F \lambda^{-1} y=g$ and $\lambda^{-1} y \geqslant 0$ which implies that $\lambda^{-1} x$ is in $\rho \operatorname{CUT}(n)$. Thus $\rho^{-1} \lambda^{-1} x$ is in $\operatorname{CUT}(n)$ and $x$ is thus a positive combination of cut-vectors, hence $x \in \operatorname{CUT}-\operatorname{CONE}(n)$. This yields $K \subseteq \mathrm{CUT}-\mathrm{CONE}(n)$. In conclusion, $K=\mathrm{CUT}-\mathrm{CONE}(n)$ and (3) is an EF of the cut cone. The size of this EF is at most $r+1$, where $r$ denotes the size of the given $\rho$-approximate EF of CUT $(n)$. Thus $\mathrm{xc}(\operatorname{CUT}-\mathrm{CONE}(n)) \leqslant r+1$.

By using the correlation mapping (see [Laurent and Deza, 1997, p. 55]), the cut cone has the same extension complexity as its corresponding correlation cone, defined as

$$
\operatorname{COR}-\operatorname{CONE}(n-1):=\operatorname{cone}\left(\left\{\left(\begin{array}{c}
b_{0} \\
b
\end{array}\right)\left(\begin{array}{c}
b_{0} \\
b
\end{array}\right)^{\top} \mid b_{0} \in\{0,1\}, b \in\{0,1\}^{n-2}\right\}\right) .
$$

We claim that the unique disjointness matrix on $[n-2]$ can be embedded in a slack matrix of $\operatorname{COR}-\operatorname{CONE}(n-1)$. To prove this, consider the $(n-1) \times(n-1)$ rank-1 positive semidefinite matrices

$$
T_{a}:=\left(\begin{array}{c}
-1 \\
a
\end{array}\right)\left(\begin{array}{c}
-1 \\
a
\end{array}\right)^{\top} \quad \text { and } \quad U^{b}:=\left(\begin{array}{l}
1 \\
b
\end{array}\right)\left(\begin{array}{l}
1 \\
b
\end{array}\right)^{\top}
$$

where $a, b \in\{0,1\}^{n-2}$. The Frobenius inner product $\left\langle T_{a}, z\right\rangle \geqslant 0$ of $T_{a}$ with any correlation matrix $z=\left(\begin{array}{c}b_{0} \\ b\end{array}\right)\left(\begin{array}{c}b_{0} \\ b\end{array}\right)^{\top}$ is nonnegative because both matrices are positive semidefinite. Thus $\left\langle T_{a}, z\right\rangle \geqslant 0$ is valid for all points $z \in \operatorname{COR}-\operatorname{CONE}(n-1)$, for all $a \in\{0,1\}^{n-2}$. Moreover, $\left\langle T_{a}, U^{b}\right\rangle=\left(1-a^{\top} b\right)^{2}$ for all $a, b \in\{0,1\}^{n-2}$ and thus $\left\langle T_{a}, U^{b}\right\rangle=\operatorname{UDISJ}(a, b)$ provided $a^{\top} b \in\{0,1\}$.

From what precedes, the slack of correlation matrix $U^{b}$ with respect to the valid inequality $\left\langle T_{a}, z\right\rangle \geqslant 0$ is $\operatorname{UDISJ}(a, b)$ provided $a^{\top} b \in\{0,1\}$. Therefore, $\operatorname{COR}-\operatorname{CONE}(n-1)$ has a slack matrix that contains UDISJ on $[n-2]$. Because the nonnegative rank of any matrix containing UDISJ is 
$2^{\Omega(n)}$ (this follows from [Razborov, 1992], see [Fiorini et al., 2012, Theorem 1]), we conclude that the nonnegative rank of some slack matrix of COR-CONE $(n-1)$ is $2^{\Omega(n)}$. From Theorem 1 lapplied to $P=Q=$ COR-CONE $(n-1)$, it follows that $\mathrm{xc}(\operatorname{COR}-\operatorname{CONE}(n-1))=2^{\Omega(n)}$. Thus we get

$$
r+1 \geqslant \mathrm{xc}(\operatorname{CUT}-\operatorname{CONE}(n))=\mathrm{xc}(\operatorname{COR}-\operatorname{CONE}(n-1))=2^{\Omega(n)},
$$

from which we obtain $r=2^{\Omega(n)}$. The result then follows immediately.

\section{Extension of Razborov's Lemma and Shifts of Unique Disjointness}

In the first subsection we generalize Razborov's famous lemma on the disjointness problem (see Razborov [1992] or Kushilevitz and Nisan [1997, Lemma 4.49] for the original version). In the next subsection we apply it to shift the UDISJ matrix without significantly decreasing its nonnegative rank, which will be used in later sections to obtain lower bounds on approximate extended formulations.

The main improvements to Razborov's lemma are threefold: (i) the dependence on the error parameter $\epsilon$ is made explicit; (ii) better analytical estimations are employed to improve overall strength of the statement; (iii) probabilities are generalized to expected values to homogenize the proof and yield a stronger lemma.

\subsection{Extension of Razborov's Rectangle Corruption Lemma}

Suppose that $n \equiv 3(\bmod 4)$ and let

$$
\begin{aligned}
\ell & :=\frac{n+1}{4}, \\
A & :=\left\{(a, b) \in 2^{[n]} \times 2^{[n]}|| a|=| b|=\ell,| a \cap b \mid=0\right\}, \\
B & :=\left\{(a, b) \in 2^{[n]} \times 2^{[n]}|| a|=| b|=\ell,| a \cap b \mid=1\right\} .
\end{aligned}
$$

Thus $A$ is the set of disjoint pairs of $\ell$-subsets and $B$ is the set of barely intersecting pairs of $\ell$-subsets. Furthermore, let $\mu$ be any distribution on pairs $(a, b)$ of subsets of $[n]$ that is supported on $A \cup B$ and uniform when conditioned to either $A$ or $B$.

Lemma 4. Let $n, \ell, A, B$ and $\mu$ be as above. For every nonnegative functions $f$ and $g$ defined on $2^{[n]} \times 2^{[n]}$ we introduce a random variable $X:=f(a) g(b)$. Then for every $0<\epsilon<1$ :

$$
(1-\epsilon) \mathbb{E}[X \mid A]-\mathbb{E}[X \mid B] \leqslant\|X \uparrow(A \cup B)\|_{\infty} 2^{-\frac{\epsilon^{2}}{16 \ln 2} \ell+O(\log \ell)},
$$

where the constant in the $O(\log \ell)$ is absolute, and $X \uparrow(A \cup B)$ denotes the restriction of $X$ to $A \cup B$.

Let us write $I_{C}$ for the indicator of an event $C$. In case $f$ and $g$ are both binary, $X$ is the indicator of a rectangle $R$, that is $X=I_{R}$, and (5) becomes

$$
(1-\epsilon) \mathbb{P}[R \mid A]-\mathbb{P}[R \mid B] \leqslant 2^{-\frac{\epsilon^{2}}{16 \ln 2} \ell+O(\log \ell)},
$$

which is a strengthened version of Razborov's original lemma.

For concreteness, the reader might find it helpful to imagine that $X$ is the indicator of a rectangle in the proof below. Our proof is inspired by the version in Kushilevitz and Nisan \1997, Lemma 4.49] and we adopt similar notations. 
Proof of Lemma 4 The proof is in four main steps.

Step 1: Expressing $\mathbb{E}[X \mid A]$ and $\mathbb{E}[X \mid B]$ in an alternative framework. The statement of the lemma does not depend on the actual probabilities of $A$ and $B$, hence for convenience, we fix them as

$$
\mathbb{P}[A]=\frac{3}{4} \quad \text { and } \quad \mathbb{P}[B]=\frac{1}{4} .
$$

This brings the advantage of the following alternative description of $\mu$.

Let $T=\left(T_{1}, T_{2},\{i\}\right)$ be a uniformly chosen partition of $[n]$ into two subsets $T_{1}, T_{2}$ with $2 \ell-1$ elements each and one singleton $\{i\}$. Given $T$ we choose $a$ as a uniform $\ell$-subset of $T_{1} \cup\{i\}=$ $[n] \backslash T_{2}$ and $b$ as a uniform $\ell$-subset of $T_{2} \cup\{i\}=[n] \backslash T_{1}$, independently. This defines a distribution $\mu$ that is supported on $A \cup B$, uniform when conditioned to either $A$ or $B$ and satisfies $\mathbb{P}[[] T] B=$ $\mathbb{P}[[] T] i \in a, i \in b=\mathbb{P}[[] T] i \in a \mathbb{P}[[] T] i \in b=(1 / 2)^{2}=1 / 4$ and thus $\mathbb{P}[[] T] A=1-1 / 4=3 / 4$. In particular, $\mathbb{P}[A]=3 / 4$ and $\mathbb{P}[B]=1 / 4$, as required.

We begin by rewriting $\mathbb{E}[X \mid B]$ and then $\mathbb{E}[X \mid A]$ in terms of the following functions of $T$ :

$$
\begin{aligned}
\operatorname{Row}_{0}(T) & :=\mathbb{E}[f(a) \mid T, i \notin a], & \operatorname{Row}_{1}(T) & :=\mathbb{E}[f(a) \mid T, i \in a], \\
\operatorname{Col}_{0}(T) & :=\mathbb{E}[g(b) \mid T, i \notin b], & \operatorname{Col}_{1}(T) & :=\mathbb{E}[g(b) \mid T, i \in b] .
\end{aligned}
$$

We note the following nice interpretation of $\operatorname{Row}_{0}(T)+\operatorname{Row}_{1}(T)$ and $\operatorname{Col}_{0}(T)+\operatorname{Col}_{1}(T)$, that we will use at the end of the proof:

$$
\begin{aligned}
\mathbb{E}[f(a) \mid T] & =\underbrace{\mathbb{E}[f(a) \mid T, i \in a]}_{\operatorname{Row}_{1}(T)} \cdot \underbrace{\mathbb{P}[i \in a \mid T]}_{1 / 2}+\underbrace{\mathbb{E}[f(a) \mid T, i \notin a]}_{\operatorname{Row}_{0}(T)} \cdot \underbrace{\mathbb{P}[i \notin a \mid T]}_{1 / 2} \\
& =\frac{\operatorname{Row}_{0}(T)+\operatorname{Row}_{1}(T)}{2}, \\
\mathbb{E}[g(b) \mid T] & =\frac{\operatorname{Col}_{0}(T)+\operatorname{Col}_{1}(T)}{2} .
\end{aligned}
$$

Note that: (i) the distribution of $(a, b)$ conditioned on a given $T$ is a product distribution (this local independence property is the main reason why we reinterpret the distribution $\mu$ ); (ii) the marginal distributions of $a$ conditioned on $(T, i \in a, i \in b)$ and $(T, i \in a)$ are the same (and similarly for $b$, we can remove the condition $i \in a)$. From these facts, we get

$$
\begin{aligned}
\mathbb{E}[X \mid B] & =\mathbb{E}[f(a) g(b) \mid i \in a, i \in b] \\
& =\mathbb{E}[\mathbb{E}[f(a) g(b) \mid T, i \in a, i \in b]] \\
& =\mathbb{E}[\mathbb{E}[f(a) \mid T, i \in a, i \in b] \mathbb{E}[g(b) \mid T, i \in a, i \in b]] \\
& =\mathbb{E}[\mathbb{E}[f(a) \mid T, i \in a] \mathbb{E}[g(b) \mid T, i \in b]] \\
& =\mathbb{E}\left[\operatorname{Row}_{1}(T) \operatorname{Col}_{1}(T)\right] .
\end{aligned}
$$

By similar arguments, we find

$$
\begin{aligned}
\mathbb{E}[X \mid A] & =\frac{1}{3} \mathbb{E}[f(a) g(b) \mid i \notin a, i \notin b]+\frac{1}{3} \mathbb{E}[f(a) g(b) \mid i \in a, i \notin b]+\frac{1}{3} \mathbb{E}[f(a) g(b) \mid i \notin a, i \in b] \\
& =\frac{1}{3} \mathbb{E}\left[\operatorname{Row}_{0}(T) \operatorname{Col}_{0}(T)\right]+\frac{1}{3} \mathbb{E}\left[\operatorname{Row}_{1}(T) \operatorname{Col}_{0}(T)\right]+\frac{1}{3} \mathbb{E}\left[\operatorname{Row}_{0}(T) \operatorname{Col}_{1}(T)\right] .
\end{aligned}
$$

Pick a $(2 \ell-1)$-subset $T_{2}$ of $[n]$, that we consider fixed for the time being. The marginal distributions of $a$ conditioned on the events $T_{2},\left(T_{2}, i \in a\right)$ and $\left(T_{2}, i \notin a\right)$ are the same, namely, the 
uniform distribution on the $\ell$-subsets of $[n] \backslash T_{2}$. (Note that fixing $T_{2}$ does not fix $i$, which could be any element of $[n] \backslash T_{2}$.) Thus, we get

$$
\mathbb{E}\left[f(a) \mid T_{2}, i \notin a\right]=\mathbb{E}\left[f(a) \mid T_{2}, i \in a\right]=\mathbb{E}\left[f(a) \mid T_{2}\right] .
$$

On the other hand, we have

$$
\begin{aligned}
\mathbb{E}\left[\operatorname{Row}_{0}(T) \mid T_{2}\right] & =\mathbb{E}\left[\mathbb{E}[f(a) \mid T, i \notin a] \mid T_{2}\right] \\
& =\mathbb{E}\left[\frac{\mathbb{E}\left[f(a) I_{i \notin a} \mid T\right]}{\mathbb{P}[i \notin a \mid T]} \mid T_{2}\right] \\
& =2 \mathbb{E}\left[f(a) I_{i \notin a} \mid T_{2}\right] \\
& =\mathbb{E}\left[f(a) \mid T_{2}, i \notin a\right]
\end{aligned}
$$

and similarly

$$
\mathbb{E}\left[\operatorname{Row}_{1}(T) \mid T_{2}\right]=\mathbb{E}\left[f(a) \mid T_{2}, i \in a\right]
$$

From (8), we conclude

$$
\mathbb{E}\left[\operatorname{Row}_{0}(T) \mid T_{2}\right]=\mathbb{E}\left[\operatorname{Row}_{1}(T) \mid T_{2}\right]
$$

Therefore (letting $T_{2}$ vary),

$$
\begin{aligned}
\mathbb{E}\left[\operatorname{Row}_{1}(T) \operatorname{Col}_{0}(T)\right] & =\mathbb{E}\left[\mathbb{E}\left[\operatorname{Row}_{1}(T) \operatorname{Col}_{0}(T) \mid T_{2}\right]\right] \\
& =\mathbb{E}\left[\mathbb{E}\left[\operatorname{Row}_{1}(T) \mid T_{2}\right] \operatorname{Col}_{0}(T)\right] \\
& =\mathbb{E}\left[\mathbb{E}\left[\operatorname{Row}_{0}(T) \mid T_{2}\right] \operatorname{Col}_{0}(T)\right] \\
& =\mathbb{E}\left[\mathbb{E}\left[\operatorname{Row}_{0}(T) \operatorname{Col}_{0}(T) \mid T_{2}\right]\right] \\
& =\mathbb{E}\left[\operatorname{Row}_{0}(T) \operatorname{Col}_{0}(T)\right] .
\end{aligned}
$$

The second and fourth equalities above are due to the fact that $\operatorname{Col}_{0}(T)$ is constant when $T_{2}$ is fixed. This is because $\operatorname{Col}_{0}(T)=\mathbb{E}[g(b) \mid T, i \notin b]$ depends only on $T_{2}$, as the marginal distribution of $b$ given $(T, i \notin b)$ is uniform on the $\ell$-subsets of $T_{2}$.

Exchanging the roles of rows and columns, we have

$$
\mathbb{E}\left[\operatorname{Row}_{1}(T) \operatorname{Col}_{0}(T)\right]=\mathbb{E}\left[\operatorname{Row}_{0}(T) \operatorname{Col}_{0}(T)\right] .
$$

In conclusion, we find the following simple expression for $\mathbb{E}[X \mid A]$ :

$$
\mathbb{E}[X \mid A]=\mathbb{E}\left[\operatorname{Row}_{0}(T) \operatorname{Col}_{0}(T)\right] .
$$

Step 2: Estimation of $\mathbb{E}[X \mid A]-\mathbb{E}[X \mid B]$. Via obvious estimates:

$$
\begin{aligned}
& \operatorname{Row}_{0}(T) \operatorname{Col}_{0}(T)-\operatorname{Row}_{1}(T) \operatorname{Col}_{1}(T) \\
& \leqslant \operatorname{Row}_{0}(T) \operatorname{Col}_{0}(T)-\min \left\{\operatorname{Row}_{0}(T), \operatorname{Row}_{1}(T)\right\} \cdot \min \left\{\operatorname{Col}_{0}(T), \operatorname{Col}_{1}(T)\right\} \\
&= \operatorname{Row}_{0}(T)\left(\operatorname{Col}_{0}(T)-\min \left\{\operatorname{Col}_{0}(t), \operatorname{Col}_{1}(T)\right\}\right) \\
& \quad+\left(\operatorname{Row}_{0}(T)-\min \left\{\operatorname{Row}_{0}(t), \operatorname{Row}_{1}(T)\right\}\right) \min \left\{\operatorname{Col}_{0}(t), \operatorname{Col}_{1}(T)\right. \\
& \leqslant \operatorname{Row}_{0}(T)\left|\operatorname{Col}_{0}(T)-\operatorname{Col}_{1}(T)\right|+\left|\operatorname{Row}_{0}(T)-\operatorname{Row}_{1}(T)\right| \operatorname{Col}_{0}(T) .
\end{aligned}
$$

This argument is depicted on Figure 2. 


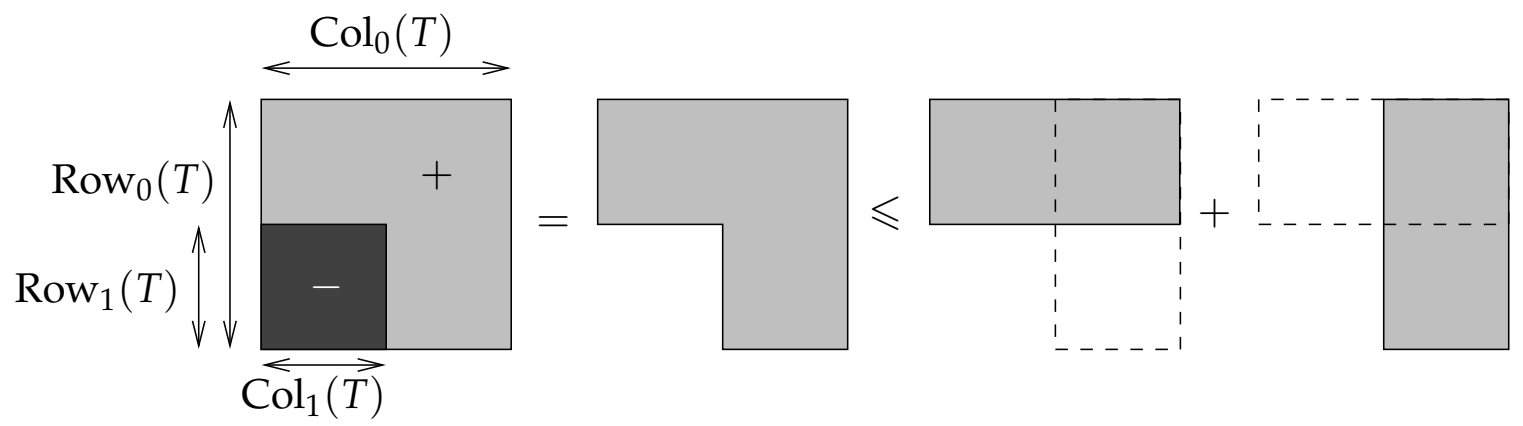

Figure 2: The estimation of $\operatorname{Row}_{0}(T) \operatorname{Col}_{0}(T)-\operatorname{Row}_{1}(T) \operatorname{Col}_{1}(T)$.

In Step 3 below, we will define two events, row-big $(T)$ and column-big $(T)$. The event small $(T)$ holds if and only if not both of row-big $(T)$ and column-big $(T)$ hold. Thus

$$
1=I_{\text {row-big }(T) \cap \operatorname{column}-\operatorname{big}(T)}+I_{\text {small }(T)} \text {. }
$$

From (11),

$$
\begin{aligned}
& \left(\operatorname{Row}_{0}(T) \operatorname{Col}_{0}(T)-\operatorname{Row}_{1}(T) \operatorname{Col}_{1}(T)\right) \cdot I_{\text {row-big}}(T) \cap \operatorname{ncolumn-big}(T) \\
& \leqslant\left(\operatorname{Row}_{0}(T)\left|\operatorname{Col}_{0}(T)-\operatorname{Col}_{1}(T)\right|+\left|\operatorname{Row}_{0}(T)-\operatorname{Row}_{1}(T)\right| \operatorname{Col}_{0}(T)\right) \cdot I_{\text {row-big}}(T) \cap \operatorname{column-big}(T) \\
& \leqslant \operatorname{Row}_{0}(T)\left|\operatorname{Col}_{0}(T)-\operatorname{Col}_{1}(T)\right| \cdot I_{\text {column-big}(T)}+\left|\operatorname{Row}_{0}(T)-\operatorname{Row}_{1}(T)\right| \operatorname{Col}_{0}(T) \cdot I_{\text {row-big }(T)} .
\end{aligned}
$$

Moreover, we obviously have

$$
\left(\operatorname{Row}_{0}(T) \operatorname{Col}_{0}(T)-\operatorname{Row}_{1}(T) \operatorname{Col}_{1}(T)\right) \cdot I_{\text {small }(T)} \leqslant \operatorname{Row}_{0}(T) \operatorname{Col}_{0}(T) \cdot I_{\text {small }(T)} .
$$

Below, we will prove

$$
\begin{aligned}
\mathbb{E}\left[\operatorname{Row}_{0}(T)\left|\operatorname{Col}_{0}(T)-\operatorname{Col}_{1}(T)\right| \cdot I_{\text {column-big }(T)}\right] & \leqslant \frac{\epsilon}{2} \mathbb{E}\left[\operatorname{Row}_{0}(T) \operatorname{Col}_{0}(T)\right], \\
\mathbb{E}\left[\left|\operatorname{Row}_{0}(T)-\operatorname{Row}_{1}(T)\right| \operatorname{Col}_{0}(T) \cdot I_{\text {row-big }(T)}\right] & \leqslant \frac{\epsilon}{2} \mathbb{E}\left[\operatorname{Row}_{0}(T) \operatorname{Col}_{0}(T)\right], \text { and } \\
\mathbb{E}\left[\operatorname{Row}_{0}(T) \operatorname{Col}_{0}(T) \cdot I_{\text {small }(T)}\right] & \leqslant\|X \uparrow(A \cup B)\|_{\infty} 2^{-\frac{\epsilon^{2}}{16 \ln 2}-O(\log \ell)}
\end{aligned}
$$

By (7), (10) and (12), these upper bounds imply

$$
\begin{aligned}
& \mathbb{E}[X \mid A]-\mathbb{E}[X \mid B] \\
& =\mathbb{E}\left[\operatorname{Row}_{0}(T) \operatorname{Col}_{0}(T)-\operatorname{Row}_{1}(T) \operatorname{Col}_{1}(T)\right] \\
& =\mathbb{E}\left[\left(\operatorname{Row}_{0}(T) \operatorname{Col}_{0}(T)-\operatorname{Row}_{1}(T) \operatorname{Col}_{1}(T)\right) \cdot\left(I_{\text {row-big }(T) \cap \operatorname{column}-\operatorname{big}(T)}+I_{\text {small }(T)}\right)\right] \\
& \leqslant 2 \frac{\epsilon}{2} \mathbb{E}\left[\operatorname{Row}_{0}(T) \operatorname{Col}_{0}(T)\right]+\|X \uparrow(A \cup B)\|_{\infty} 2^{-\frac{\epsilon^{2}}{16 \ln 2} \ell-O(\log \ell)} \\
& =\epsilon \mathbb{E}[X \mid A]+\|X \uparrow(A \cup B)\|_{\infty} 2^{-\frac{\epsilon^{2}}{16 \ln 2} \ell-O(\log \ell)}
\end{aligned}
$$

from which the result clearly follows, by rearranging.

Step 3. One-sided error estimation via entropy argument in the "big" case. Let $\delta>0$ be a constant to be chosen later. Essentially, $\delta$ will be the coefficient of $\ell$ in the exponent. Let row-big $(T)$ denote the 


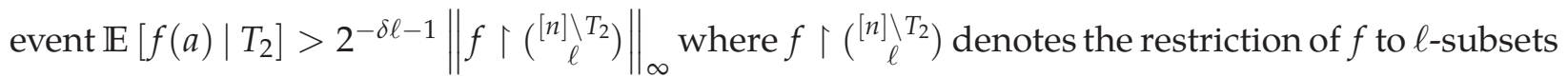
of $[n] \backslash T_{2}$. The event column-big $(T)$ is defined in a similar way. These events depend only on $T_{2}$ and $T_{1}$, respectively.

Let $T_{2}$ be fixed and assume that row-big $(T)$ holds. In particular $\mathbb{E}\left[f(a) \mid T_{2}\right]$ is positive. Because $\left(\begin{array}{c}2 \ell-1 \\ \ell-1\end{array}\right)=\left(\begin{array}{c}2 \ell-1 \\ \ell\end{array}\right)$, the distribution of $a$ given $T_{2}$ is the same as the distribution of $a$ given $T$, for every fixed choice of $i$. Thus, we have

$$
\mathbb{E}[f(a) \mid T]=\mathbb{E}\left[f(a) \mid T_{2}\right]=\sum_{\substack{x \subseteq[n] \backslash T_{2} \\
|x|=\ell}} \frac{1}{\left(\begin{array}{c}
2 \ell \\
\ell
\end{array}\right)} f(x) .
$$

(This holds when $f(a)$ is replaced by any function of $a$.)

We define $s$ as a random $\ell$-subset of $[n] \backslash T_{2}$ with distribution

$$
\mathbb{P}\left[s=x \mid T_{2}\right]=\frac{f(x)}{\left(\begin{array}{c}
2 \ell \\
\ell
\end{array}\right) \mathbb{E}\left[f(a) \mid T_{2}\right]}=\frac{f(x)}{\sum_{\substack{y \subseteq[n] \backslash T_{2} \\
|y|=\ell}} f(y)} \leqslant \frac{2^{\delta \ell+1}}{\left(\begin{array}{c}
2 \ell \\
\ell
\end{array}\right)} .
$$

Let us introduce the shorthand notation $\lambda:=\mathbb{P}\left[i \in s \mid T_{2}\right]$. Then

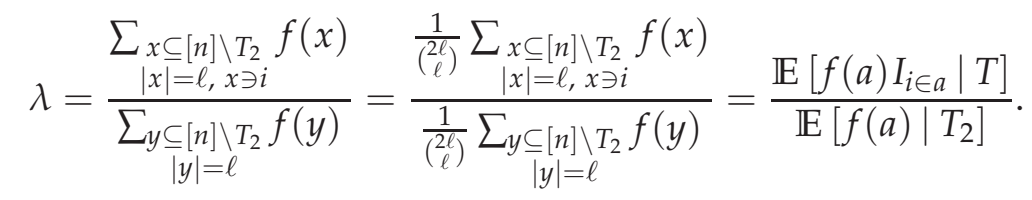

Hence,

$$
\begin{aligned}
& \operatorname{Row}_{1}(T)=2 \mathbb{E}\left[f(a) I_{i \in a} \mid T\right]=2 \mathbb{E}\left[f(a) \mid T_{2}\right] \cdot \mathbb{P}\left[i \in s \mid T_{2}\right]=2 \lambda \mathbb{E}\left[f(a) \mid T_{2}\right], \\
& \operatorname{Row}_{0}(T)=2 \mathbb{E}\left[f(a) I_{i \notin a} \mid T\right]=2 \mathbb{E}\left[f(a) \mid T_{2}\right] \cdot \mathbb{P}\left[i \notin s \mid T_{2}\right]=2(1-\lambda) \mathbb{E}\left[f(a) \mid T_{2}\right] .
\end{aligned}
$$

We now estimate the entropy of $s$. On the one hand, by subadditivity of the entropy, we get the following upperbound on $H\left(s \mid T_{2}\right)$ :

$$
H\left(s \mid T_{2}\right) \leqslant \sum_{j \in[n] \backslash T_{2}} H\left(I_{j \in s} \mid T_{2}\right)=2 \ell \mathbb{E}\left[H(\lambda) \mid T_{2}\right] .
$$

In this last equation, $H(\lambda)$ denotes the binary entropy of $\lambda$. On the other hand, we get a lower bound on $H\left(s \mid T_{2}\right)$ from our upper bound on the distribution of $s$ (which induces "flatness" of the distribution):

$$
\begin{aligned}
H\left(s \mid T_{2}\right) & =\sum_{x} \mathbb{P}\left[s=x \mid T_{2}\right] \log \frac{1}{\mathbb{P}\left[s=x \mid T_{2}\right]} \\
& \geqslant \sum_{x} \mathbb{P}\left[s=x \mid T_{2}\right] \log \frac{\left(\begin{array}{c}
2 \ell \\
\ell
\end{array}\right)}{2^{\delta \ell+1}}=\log \frac{\left(\begin{array}{c}
2 \ell \\
\ell
\end{array}\right)}{2^{\delta \ell+1}}=2 \ell\left(1-\frac{\delta}{2}-O\left(\frac{\log \ell}{\ell}\right)\right) .
\end{aligned}
$$

This implies

$$
\frac{\delta}{2}+O\left(\frac{\log \ell}{\ell}\right) \geqslant \mathbb{E}\left[1-H(\lambda) \mid T_{2}\right]
$$

To estimate this expression, we use the Taylor expansion of the binary entropy function at $1 / 2$ :

$$
1-H(x) \geqslant \frac{(1-2 x)^{2}}{2 \ln 2} .
$$


Hence (18) yields

$$
\frac{\delta}{2}+O\left(\frac{\log \ell}{\ell}\right) \geqslant \frac{\mathbb{E}\left[(1-2 \lambda)^{2} \mid T_{2}\right]}{2 \ln 2} \geqslant \frac{\left(\mathbb{E}\left[|1-2 \lambda| \mid T_{2}\right]\right)^{2}}{2 \ln 2}
$$

From (8), (9) we have $\mathbb{E}\left[f(a) \mid T_{2}\right]=\mathbb{E}\left[\operatorname{Row}_{0}(T) \mid T_{2}\right]$. Using (17) and (16), we derive

$$
\begin{aligned}
\mathbb{E}\left[\left|\operatorname{Row}_{0}(T)-\operatorname{Row}_{1}(T)\right| \mid T_{2}\right] & =\mathbb{E}\left[\left|2(1-\lambda) \mathbb{E}\left[f(a) \mid T_{2}\right]-2 \lambda \mathbb{E}\left[f(a) \mid T_{2}\right]\right| \mid T_{2}\right] \\
& =2 \mathbb{E}\left[|1-2 \lambda| \mid T_{2}\right] \mathbb{E}\left[f(a) \mid T_{2}\right] \\
& \leqslant 2 \sqrt{\delta^{\prime}} \mathbb{E}\left[\operatorname{Row}_{0}(T) \mid T_{2}\right] .
\end{aligned}
$$

with

$$
\delta^{\prime}:=\left(\delta+O\left(\frac{\log \ell}{\ell}\right)\right) \ln 2
$$

We now globalize to prove (14):

$$
\begin{aligned}
& \mathbb{E}\left[\left|\operatorname{Row}_{0}(T)-\operatorname{Row}_{1}(T)\right| \operatorname{Col}_{0}(T) I_{\text {row-big }(T)}\right] \\
& =\mathbb{E}\left[\mathbb{E}\left[\left|\operatorname{Row}_{0}(T)-\operatorname{Row}_{1}(T)\right| \operatorname{Col}_{0}(T) I_{\text {row-big }(T)} \mid T_{2}\right]\right] \\
& =\mathbb{E}\left[\mathbb{E}\left[\left|\operatorname{Row}_{0}(T)-\operatorname{Row}_{1}(T)\right| I_{\text {row-big }(T)} \mid T_{2}\right] \operatorname{Col}_{0}(T)\right] \\
& \leqslant \mathbb{E}\left[2 \sqrt{\delta^{\prime}} \mathbb{E}\left[\operatorname{Row}_{0}(T) \mid T_{2}\right] \operatorname{Col}_{0}(T)\right] \\
& =2 \sqrt{\delta^{\prime}} \mathbb{E}\left[\operatorname{Row}_{0}(T) \operatorname{Col}_{0}(T)\right]
\end{aligned}
$$

We require $\frac{\epsilon}{2}=2 \sqrt{\delta^{\prime}}$, from which we express $\delta$ in terms of $\epsilon$ using (19):

$$
\delta=\frac{\delta^{\prime}}{\ln 2}-O\left(\frac{\log \ell}{\ell}\right)=\frac{\epsilon^{2}}{16 \ln 2}-O\left(\frac{\log \ell}{\ell}\right)
$$

This concludes the proof of (14). Equation (13) follows by exchanging rows and columns.

Step 4: Error estimation in the "small" case. Suppose that for some given $T$, small $(T)$ holds because row-big $(T)$ does not hold (the argument is similar in case column-big $(T)$ does not hold). Then, using (6),

$$
\operatorname{Row}_{0}(T) \leqslant \operatorname{Row}_{0}(T)+\operatorname{Row}_{1}(T)=2 \mathbb{E}[f(a) \mid T] .
$$

Thus

$$
\begin{aligned}
\operatorname{Row}_{0}(T) \operatorname{Col}_{0}(T) & \leqslant 2 \mathbb{E}[f(a) \mid T] \cdot \mathbb{E}[g(b) \mid T, i \notin b] \\
& \leqslant 2^{-\delta \ell}\left\|f(a) \uparrow\left(\begin{array}{c}
{[n] \backslash T_{2}} \\
\ell
\end{array}\right)\right\|_{\infty} \cdot\left\|g(b) \uparrow\left(\begin{array}{c}
T_{2} \\
\ell
\end{array}\right)\right\|_{\infty} \\
& \leqslant 2^{-\delta \ell}\|f(a) g(b) \uparrow(A \cup B)\|_{\infty}
\end{aligned}
$$

This is easily seen to imply (15). 


\subsection{Lower Bounds for Shifts of Unique Disjointness}

Now we apply Lemma 4 to show that the nonnegative rank (and hence the communication complexity in expectation) of any shifted version of the unique disjointness matrix remains high. More precisely, let $M \in \mathbb{R}_{+}^{2^{n} \times 2^{n}}$; for convenience we index the rows and columns with elements in $\{0,1\}^{n}$. We say that $M$ is a $\rho$-extension of UDISJ, if $M_{a b}=\rho$ whenever $|a \cap b|=0$ and $M_{a b}=\rho-1$ whenever $|a \cap b|=1$ with $a, b \in\{0,1\}^{n}$. Note that for these pairs $M$ has exclusively positive entries whenever $\rho>1$. For $\rho=1$ a nonnegative rank of $2^{\Omega(n)}$ was already shown in Fiorini et al. [2012] via nondeterministic communication complexity. We now extend this result for a wide range of $\rho$ using Lemma 4 ,

Theorem 5 (Nonnegative rank of UDISJ shifts). Let $M \in \mathbb{R}_{+}^{2^{n} \times 2^{n}}$ be a $\rho$-extension of UDISJ as above.

(i) If $\rho$ is a fixed constant, then $\operatorname{rank}_{+}(M)=2^{\Omega(n)}$.

(ii) If $\rho=O\left(n^{\beta}\right)$ for some constant $\beta<1 / 2$ then $\operatorname{rank}_{+}(M)=2^{\Omega\left(n^{1-2 \beta}\right)}$.

Proof. Without loss of generality, assume $n \equiv 3(\bmod 4)$. Let $r=\operatorname{rank}_{+}(M)$. Regarding the $2^{n} \times 2^{n}$ matrix $M$ as a function from $2^{[n]} \times 2^{[n]}$ to $\mathbb{R}$, we can write $M=\sum_{i=1}^{r} X_{i}$ where $X_{i}(a, b)=$ $f_{i}(a) g_{i}(b)$ for some nonnegative functions $f_{i}$ and $g_{i}$ defined over $2^{[n]}$. Then

$$
\mathbb{E}[M \mid A]=\rho \quad \text { and } \quad \mathbb{E}[M \mid B]=\rho-1 .
$$

On the other hand, by applying Lemma 4 to each $i \in[r]$ and summing up all equations we find

$$
\begin{aligned}
(1-\epsilon) \mathbb{E}[M \mid A]-\mathbb{E}[M \mid B] & \leqslant \sum_{i=1}^{r}\left\|X_{i} \uparrow(A \cup B)\right\|_{\infty} 2^{-\frac{\epsilon^{2}}{16 \ln 2} \ell+O(\log \ell)} \\
& \leqslant r\|M \uparrow(A \cup B)\|_{\infty} 2^{-\frac{\epsilon^{2}}{16 \ln 2} \ell+O(\log \ell)}
\end{aligned}
$$

where $\ell=\frac{n+1}{4}$ as before. By plugging in the values of $\mathbb{E}[M \mid A], \mathbb{E}[M \mid B]$ and $\|M \uparrow(A \cup B)\|_{\infty}$, we get

$$
(1-\epsilon) \rho-\rho+1 \leqslant r \cdot \rho \cdot 2^{-\frac{\epsilon^{2}}{16 \ln 2} \ell+O(\log \ell)},
$$

which provides the lower bound

$$
r \geqslant\left(\frac{1}{\rho}-\epsilon\right) 2 \frac{\epsilon^{2}}{16 \ln 2} \ell-O(\log \ell) .
$$

If $\rho$ is constant, this last expression is $2^{\Omega(n)}$ provided $\epsilon$ is chosen sufficiently close to 0 . This proves part (i) of the theorem.

If $\rho \leqslant \widehat{C n^{\beta}}$ for some positive constant $C$, then we can take $\epsilon=\frac{1}{2 \mathrm{Cn}^{\beta}}$. Thus $\frac{1}{\rho}-\epsilon \geqslant \frac{1}{2 \mathrm{Cn}^{\beta}}=$ $\Omega\left(n^{-\beta}\right)$. This leads to the lower bound $r \geqslant 2^{\Omega\left(n^{1-2 \beta}\right)}$ as claimed in part (ii).

\section{Polyhedral Inapproximability of CLIQUE and SDPs}

We will now use Theorem 5 in combination with Theorem 2 to lower bound the sizes of approximate EFs for CLIQUE and some SDPs. First, we pinpoint a pair $P, Q$ of nested polyhedra that will be the source of our polyhedral inapproximability results. Second, we give a faithful linear encoding of CLIQUE and prove strong lower bounds on the sizes of approximate EFs for CLIQUE w.r.t. this encoding. Third, we focus on approximations of SDPs by LPs. 


\subsection{A Hard Pair}

Let $n$ be a positive integer. The correlation polytope $\operatorname{COR}(n)$ is defined as the convex hull of all the $n \times n$ rank- 1 binary matrices of the form $b b^{\top}$ where $b \in\{0,1\}^{n}$. In other words,

$$
\operatorname{COR}(n)=\operatorname{conv}\left(\left\{b b^{\top} \mid b \in\{0,1\}^{n}\right\}\right) .
$$

This will be our inner polytope $P$. Next, let

$$
Q=Q(n):=\left\{x \in \mathbb{R}^{n \times n} \mid\left\langle 2 \operatorname{diag}(a)-a a^{\top}, x\right\rangle \leqslant 1, a \in\{0,1\}^{n}\right\},
$$

where $\langle\cdot, \cdot\rangle$ denotes the Frobenius inner product. This will be our outer polyhedron $Q$.

Then the following is known, see [Fiorini et al., 2012]. First, $P \subseteq Q$. Second, denoting by $S^{P, Q}$ the slack matrix of the pair $P, Q$, we have $S_{a b}^{P, Q}=\left(1-a^{\top} b\right)^{2}$. Thus, for $\rho \geqslant 1$, we have $S_{a b}^{P, \rho Q}=$ $\left(1-a^{\top} b\right)^{2}+\rho-1$. Observe that the matrix $S^{P, \rho Q}$ is a $\rho$-extension of UDISJ and therefore has high nonnegative rank via Theorem 5; moreover it has positive entries everywhere for $\rho>1$. Together with Theorem 1 this implies that every polyhedron sandwiched between $P=\operatorname{COR}(n)$ and $\rho Q$ has large extension complexity. We obtain the following theorem.

Theorem 6 (Lower bounds for approximate EFs of the hard pair). Let $\rho \geqslant 1$, let $n$ be a positive integer and let $P=\operatorname{COR}(n), Q=Q(n)$ be as above. Then the following hold:

(i) If $\rho$ is a fixed constant, then $\operatorname{xc}(P, \rho Q)=2^{\Omega(n)}$.

(ii) If $\rho=O\left(n^{\beta}\right)$ for some constant $\beta<1 / 2$, then $\mathrm{xc}(P, \rho Q)=2^{\Omega\left(n^{1-2 \beta}\right)}$.

\subsection{Polyhedral Inapproximability of CLIQUE}

We define a natural linear encoding for the maximum clique problem (CLIQUE) as follows. Let $n$ denote the number of vertices of the input graph. We define a $d=n^{2}$ dimensional encoding. The variables are denoted by $x_{i j}$ for $i, j \in[n]$. Thus $x \in \mathbb{R}^{n \times n}$. The interpretation is that a set of vertices $X$ is encoded by $x_{i j}=1$ if $i, j \in X$ and $x_{i j}=0$ otherwise. Note that $X=\left\{i: x_{i i}=1\right\}$ can be recovered from only the diagonal variables. This defines the set $\mathcal{L} \subseteq\{0,1\}^{*}$ of feasible solutions. Notice that $x \in\{0,1\}^{n \times n}$ is feasible if and only if it is of the form $x=b b^{\top}$ for some $b \in\{0,1\}^{n}$, the characteristic vector of $X$. Thus we have $P=\operatorname{COR}(n)$ for the inner polytope.

The admissible objective functions are chosen as follows to encode the CLIQUE problem for graphs $G$ supported on $[n]$. Given a graph $G$ such that $V(G) \subseteq[n]$, we let $w_{i i}:=1$ for $i \in V(G)$, $w_{i i}:=0$ for $i \in[n] \backslash V(G), w_{i j}=w_{j i}:=-1$ when $i j$ is a non-edge of $G$ (that is, $i, j \in V(G), i \neq j$ and $i j \notin E(G))$, and $w_{i j}=w_{j i}:=0$ otherwise. We denote the resulting weight vector by $w^{G}$. Notice that for a graph $G$ with $V(G)=[n]$, we have $w^{G}=I-A(\bar{G})$ where $I$ is the $n \times n$ identity matrix, $A(\bar{G})$ is the adjacency matrix of the complement of $G$.

A feasible solution $x=b b^{\top} \in\{0,1\}^{n \times n}$ maximizes $\left\langle w^{G}, x\right\rangle$ only if $b$ is the characteristic vector (or incidence vector) of a clique of $G$. Indeed, if $b=\chi^{X}$ and $i j$ is a non-edge of $G$ with $i, j \in X$ then removing $i$ or $j$ from $X$ increases $\left\langle w^{G}, x\right\rangle$. Moreover, the maximum of $\left\langle w^{G}, x\right\rangle$ over $x \in\{0,1\}^{n \times n}$ feasible is the clique number $\omega(G)$.

The admissible objective functions are the ones of the form $w^{G}$, i.e., $\mathcal{O}=\left\{w^{G}: V(G) \subseteq[n]\right\}$ is the set of admissible functions. Therefore, $(\mathcal{L}, \mathcal{O})$ defines a valid linear encoding of CLIQUE. We denote the outer convex set of this linear encoding by $Q^{\text {all }}$. It is actually the polyhedron defined as $Q^{\text {all }}=\left\{x \in \mathbb{R}^{n \times n} \mid \forall\right.$ graphs $G$ s.t. $\left.V(G) \subseteq[n]:\left\langle w^{G}, x\right\rangle \leqslant \omega(G), \forall i \neq j \in[n]: x_{i j} \geqslant 0\right\}$. We will now show that $Q^{\text {all }} \subseteq Q$.

Lemma 7. Let $Q^{\text {all }}, Q$ be as above, then $Q^{\text {all }} \subseteq Q$. 
Proof. Let $x \in Q^{\text {all }}$. We want to prove that $x$ satisfies all the constraints defining $Q$. We show this by restricting to graphs $G$ with $\omega(G)=1$. For a given $a \in\{0,1\}^{n}$, let $G$ be the graph with $\chi^{V(G)}=a$ and $E(G)=\varnothing$. Then,

$$
\left\langle 2 \operatorname{diag}(a)-a a^{T}, x\right\rangle=\left\langle w^{G}, x\right\rangle \leqslant \omega(G)=1 .
$$

The lemma follows.

Because $Q^{\text {all }}$ is contained in the polyhedron $Q$ defined above, every $K$ satisfying $P \subseteq K \subseteq \rho Q^{\text {all }}$ also satisfies $P \subseteq K \subseteq \rho Q$. Hence, Theorem 6 yields the following result.

Theorem 8 (Polyhedral inapproximability of CLIQUE). W.r.t. the linear encoding defined above, CLIQUE has an $O\left(n^{2}\right)$-size $n$-approximate EF. Moreover, every $n^{1 / 2-\epsilon}$-approximate EF of CLIQUE has size $2^{\Omega\left(n^{2 \epsilon}\right)}$, for all $0<\epsilon<1 / 2$.

Proof. The $n$-approximate EF of CLIQUE is trivial: it is defined by the system $0 \leqslant x \leqslant 1$, or in slack form $x-y=0, x+z=\mathbf{1}, y \geqslant 0, z \geqslant 0$. We claim that this defines a $n$-approximate EF of CLIQUE of size $2 n^{2}$. Indeed, letting $K=[0,1]^{n \times n}$ denote the polytope defined by this EF, we have $P \subseteq K$. Moreover, $\max \{\langle w, x\rangle \mid x \in K\} \leqslant n \leqslant n \cdot \max \{\langle w, x\rangle \mid x \in P\}$ for all admissible objective functions $w$ of dimension $n \times n$ with a nonzero diagonal. In case an admissible $w$ has $w_{i i}=0$ for all $i \in[n]$, we have $\max \{\langle w, x\rangle \mid x \in K\}=0=\max \{\langle w, x\rangle \mid x \in P\}$. Our claim and the first part of the theorem follows.

The second part of the theorem follows directly from Theorem 6 and the fact that $Q^{\text {all }} \subseteq Q$.

\subsection{Polyhedral Inapproximability of SDPs}

In this section we show that there exists a spectrahedron with small semidefinite extension complexity but high approximate extension complexity; i.e., any sufficiently fine polyhedral approximation is large. This indicates that in general it is not possible to approximate SDPs arbitrarily well using small LPs, so that SDPs are indeed a much stronger class of optimization problems. (The situation looks quite different for SOCPs, see Ben-Tal and Nemirovski [2001].) The result follows from Theorem 6 and Fiorini et al. [2012].

We denote the vector space of all $r \times r$ symmetric matrices by $\mathbb{S}^{r}$ and the cone of all $r \times r$ symmetric positive semidefinite matrices (shortly, the PSD cone) by $\mathbb{S}_{+}^{r}$. A semidefinite EF of a convex set $S \subseteq \mathbb{R}^{d}$ is a linear system $\left\langle E_{i}, x\right\rangle+\left\langle F_{i}, Y\right\rangle=g_{i}(i \in[k]), Y \in \mathbb{S}_{+}^{r}$ where $E_{i} \in \mathbb{R}^{d}$ and $F_{i} \in \mathbb{S}^{r}$, such that $x \in S$ if and only if $\exists Y \in \mathbb{S}_{+}^{r}$ with $\left\langle E_{i}, x\right\rangle+\left\langle F_{i}, Y\right\rangle=g_{i}$ for all $i \in[m]$. Thus a convex set admits a semidefinite EF if and only if it is a spectrahedron. The size of the semidefinite EF $\left\langle E_{i}, x\right\rangle+\left\langle F_{i}, Y\right\rangle=g_{i}(i \in[k]), Y \in \mathbb{S}_{+}^{r}$ is simply $r$. The semidefinite extension complexity of a spectrahedron $S \subseteq \mathbb{R}^{d}$ is the minimum size of a semidefinite EF of $S$. This is denoted by $\operatorname{xC}_{S D P}(S)$. A rank-r PSD-factorization of a nonnegative matrix $M \in \mathbb{R}^{m \times n}$ is given by matrices $T_{1}, \ldots, T_{m} \in \mathbb{S}_{+}^{r}$ and $U^{1}, \ldots, U^{n} \in \mathbb{S}_{+}^{r}$, so that $M_{i j}=\left\langle T_{i}, U^{j}\right\rangle$; the PSD-rank of $M$ is the smallest $r$ such that there exists such a factorization. Yannakakis's factorization theorem can be generalized to the SDP-case (see Gouveia et al. [2013a]), i.e., the semidefinite extension complexity of a pair of polyhedra $P, Q$ equals the PSD-rank of any associated slack matrix, in most cases (e.g., Gouveia et al. [2013b] prove the equality under the hypothesis that $Q$ does not contain any line).

Let $P=\operatorname{COR}(n)$ be the correlation polytope and $Q=Q(n) \subseteq \mathbb{R}^{n \times n}$ be the polyhedron defined above in Section 4.1. Although every polyhedron $K$ sandwiched between $P$ and $Q$ has superpolynomial extension complexity, and by Theorem 6 this even applies to polyhedra sandwiched between $P$ and $\rho Q$ for $\rho=O\left(n^{1 / 2-\epsilon}\right)$, there exists a spectrahedron $S$ sandwiched between $P$ and $Q$ with small semidefinite extension complexity. 
Lemma 9 (Existence of spectrahedron). Let $n$ be a positive integer and let $P=\operatorname{COR}(n), Q=Q(n)$ be as above. Then there exists a spectrahedron $S$ in $\mathbb{R}^{n \times n}$ with $P \subseteq S \subseteq Q$ and $\operatorname{xc}_{S D P}(S) \leqslant n+1$.

Proof. For $a, b \in\{0,1\}^{n}$, the matrices $T^{a}, U_{b} \in \mathbb{S}_{+}^{n+1}$ defined in (4) satisfy $\left\langle T^{a}, U_{b}\right\rangle=\left(1-a^{\top} b\right)^{2}$. Let $M=M(n) \in \mathbb{R}^{2^{n} \times 2^{n}}$ be the matrix defined as $M_{a b}=\left(1-a^{\top} b\right)^{2}$. The matrix $M$ is an $O\left(n^{2}\right)$-rank nonnegative matrix extending the UDISJ matrix, and also the slack matrix of the pair $P, Q$. Then $M_{a b}=\left\langle T_{a}, U^{b}\right\rangle$ is a rank- $(n+1)$ PSD-factorization of $M$.

Consider the system

$$
\left\langle 2 \operatorname{diag}(a)-a a^{\top}, x\right\rangle+\left\langle T_{a}, Y\right\rangle=1 \quad\left(a \in\{0,1\}^{n}\right), \quad Y \in \mathbb{S}_{+}^{n+1}
$$

and $S$ be the projection to $\mathbb{R}^{n \times n}$ of the pairs $(x, Y) \in \mathbb{R}^{n \times n} \times \mathbb{S}^{n+1}$ satisfying (20).

First observe that $S \subseteq Q$ : since $T_{a} \in \mathbb{S}_{+}^{n+1}$ for all $a \in\{0,1\}^{n}$ and $Y \in \mathbb{S}_{+}^{n+1}$ we have $\left\langle T_{a}, Y\right\rangle \geqslant 0$ and thus $\left\langle 2 \operatorname{diag}(a)-a a^{\top}, x\right\rangle \leqslant 1$ holds for all $x \in S$.

In order to show that $P \subseteq S$ recall that $M$ is the slack matrix of the pair $P, Q$. Therefore, for each vertex $x:=b b^{\top}$ of $P$, we can pick $Y:=U^{b}$ from the factorization such that $\left\langle 2 \operatorname{diag}(a)-a a^{\top}, x\right\rangle+$ $\left\langle T_{a}, Y\right\rangle=1$ and $Y \in S_{+}^{n+1}$. It follows that $P \subseteq S$.

Our final result is the following inapproximability theorem for spectrahedra. Let us denote the closed $\varepsilon$-neighbourhood of $S$ in the $\ell_{1}$-norm by $S^{\varepsilon}:=\left\{x \in \mathbb{R}^{n \times n} \mid \exists x_{0} \in S:\left\|x-x_{0}\right\|_{1} \leqslant \varepsilon\right\}$.

Theorem 10 (Polyhedral inapproximability of SDPs). Let $\rho \geqslant 1$, and let $n$ be a positive integer. Then there exists a spectrahedron $S \subseteq \mathbb{R}^{n \times n}$ with $\operatorname{xc}_{S D P}(S) \leqslant n+1$ such that for every polyhedron $K$ with $S \subseteq K \subseteq S^{\rho-1}$ the following hold:

(i) If $\rho$ is a fixed constant, then $\mathrm{xc}(K)=2^{\Omega(n)}$.

(ii) If $\rho=O\left(n^{\beta}\right)$ for some constant $\beta<1 / 2$, then $\mathrm{xc}(K)=2^{\Omega\left(n^{1-2 \beta}\right)}$.

Proof. By Lemma 9 , there is a spectrahedron $S$ with $P \subseteq S \subseteq Q$ and $\operatorname{xcSDP}_{S D}(S) \leqslant n+1$. We now show $S^{\rho-1} \subseteq \rho Q$. Let $x \in S^{\rho-1}$, and let $x_{0} \in S$ with $\left\|x-x_{0}\right\|_{1} \leq \rho-1$. As $S \subseteq Q$, we also have $x_{0} \in Q$, hence for every $a \in\{0,1\}^{n}$ we obtain

$$
\begin{aligned}
\left\langle 2 \operatorname{diag}(a)-a a^{\top}, x\right\rangle & =\left\langle 2 \operatorname{diag}(a)-a a^{\top}, x-x_{0}\right\rangle+\left\langle 2 \operatorname{diag}(a)-a a^{\top}, x_{0}\right\rangle \\
& \leq \underbrace{\left\|2 \operatorname{diag}(a)-a a^{\top}\right\|_{\infty}}_{\leq 1} \cdot \underbrace{\left\|x-x_{0}\right\|_{1}}_{\leq \rho-1}+1 \leq \rho .
\end{aligned}
$$

Therefore $x \in \rho Q$. Therefore, $S^{\rho-1} \subseteq \rho Q$ for $\rho \geqslant 1$. If now $K$ is a polyhedron such that $S \subseteq K \subseteq$ $S^{\rho-1}$ then also $P \subseteq K \subseteq \rho Q$. The result thus follows from Theorem 6 ,

\section{Concluding Remarks}

We have introduced a general framework to study approximation limits of small LP relaxations. Given a polyhedron $Q$ encoding admissible objective functions and a polytope $P$ encoding feasible solutions, we have proved that any LP relaxation sandwiched between $P$ and a dilate $\rho Q$ has extension complexity at least the nonnegative rank of the slack matrix of the pair $P, \rho Q$.

This yields a lower bound depending only on the linear encoding of the problem at hand, and applies independently of the structure of the actual relaxation. By doing so, we obtain unconditional lower bounds on integrality gaps for small LP relaxations, which hold even in the unlikely event that $P=N P$. 
We have proved that every polynomial-size LP relaxation for (a natural linear encoding of) CLIQUE has essentially an $\Omega(\sqrt{n})$ integrality gap. As mentioned above, this was recently improved by Braverman and Moitra [2013] to a tight $\Omega\left(n^{1-\epsilon}\right)$ integrality gap, see Braun and Pokutta [2013] for a short proof and many generalizations.

Finally, our work sheds more light on the inherent limitations of LPs in the context of combinatorial optimization and approximation algorithms, in particular, in comparison to SDPs. We provide strong evidence that certain approximation guarantees can only be achieved via non-LPbased techniques (e.g., SDP-based or combinatorial).

Actually, our work has inspired Chan et al. [2013] to prove lower bounds on the size of LPs for approximating Max CUT, Max k-SAT and in fact any Max CSP. Among other results, they obtain a $n^{\Omega(\log n / \log \log n)}$ lower bound on the size of any $(2-\epsilon)$-approximate EF for Max CUT (of course, with nonnegative weights). Chan et al. [2013] thus proving the following conjecture on Max CUT that we stated in an earlier version of this text:

Theorem 11. Chan et al. 2013] It is not possible to approximate Max CUT with LPs of poly-size within a factor better than 2 .

This is in stark contrast with the ratio achieved by the SDP-based algorithm of Goemans and Williamson [1995] which is known to be optimal, assuming the Unique Games Conjecture Khot [2002], Khot et al. [2007], Mossel et al. [2005].

Next, about CLIQUE itself, here is an interesting question that this paper leaves open, as pointed out by one of the referees: find an $n$-vertex graph $G$ for which the clique polytope CLIQUE $(G):=$

conv $\left(\left\{\chi^{K} \in \mathbb{R}^{V(G)} \mid K \subseteq V(G)\right.\right.$ is a clique $\left.\}\right)$ has no polynomial-size $n^{1-\epsilon}$-approximate EF. Note that encoding CLIQUE through the clique polytope does not satisfy our faithfulness condition.

Finally, so far no strong lower bounding technique for semidefinite EFs are known. It is plausible that in the near future we will see lower bounding techniques on the PSD rank that would be suited for studying approximation limits of SDPs. (We remark however that such bounds should not only argue on the zero/nonzero pattern of a slack matrix.)

\section{Acknowledgements}

We would like to thank the two referees for their time and comments which contributed to improve the text.

\section{References}

S. Arora, B. Bollobás, and L. Lovász. Proving integrality gaps without knowing the linear program. In Proc. FOCS, pages 313-322, 2002.

S. Arora, S. Rao, and U. Vazirani. Expander flows, geometric embeddings and graph partitioning. J. ACM, 56(2):5, 2009.

S. Arora, R. Ge, R. Kannan, and A. Moitra. Computing a nonnegative matrix factorizationprovably. In Proceedings of the 44th symposium on Theory of Computing, pages 145-162. ACM, 2012.

Z. Bar-Yossef, T. Jayram, R. Kumar, and D. Sivakumar. An information statistics approach to data stream and communication complexity. J. Comput. System Sci., 68(4):702-732, 2004.

B. Barak, P. Raghavendra, and D. Steurer. Rounding semidefinite programming hierarchies via global correlation. In Proc. FOCS, pages 472-481. IEEE, 2011. 
B. Barak, F. G. Brandao, A. W. Harrow, J. Kelner, D. Steurer, and Y. Zhou. Hypercontractivity, sum-of-squares proofs, and their applications. In Proceedings of the 44th symposium on Theory of Computing, pages 307-326. ACM, 2012a.

B. Barak, P. Gopalan, J. Hastad, R. Meka, P. Raghavendra, and D. Steurer. Making the long code shorter. In Foundations of Computer Science (FOCS), 2012 IEEE 53rd Annual Symposium, pages 370-379. IEEE, 2012b.

A. Ben-Tal and A. Nemirovski. On polyhedral approximations of the second-order cone. Math. Oper. Res., 26:193-205, 2001.

D. Bienstock. Approximate formulations for 0-1 knapsack sets. Oper. Res. Lett., 36(3):317-320, 2008.

G. Braun and S. Pokutta. Common information and unique disjointness. In Foundations of Computer Science (FOCS), 2013 IEEE 54th Annual Symposium, pages 688-697. IEEE, 2013.

M. Braverman and A. Moitra. An information complexity approach to extended formulations. To appear in Proc. STOC, 2013.

R. D. Carr, G. Konjevod, G. Little, V. Natarajan, and O. Parekh. Compacting cuts: A new linear formulation for minimum cut. ACM Trans. Algorithms, 5(3):27:1-27:16, July 2009.

S. Chan, J. Lee, P. Raghavendra, and D. Steurer. Approximate constraint satisfaction requires large lp relaxations. In Foundations of Computer Science (FOCS), 2013 IEEE 54th Annual Symposium, pages 350-359. IEEE, 2013.

M. Charikar, K. Makarychev, and Y. Makarychev. Integrality gaps for sherali-adams relaxations. In Proceedings of the 41st annual ACM symposium on Theory of computing, pages 283-292. ACM, 2009.

M. Charikar, K. Makarychev, and Y. Makarychev. Local global tradeoffs in metric embeddings. SIAM J. Comput., 39(6):2487-2512, 2010.

A. Chattopadhyay and T. Pitassi. The story of set disjointness. SIGACT News, 41:59-85, 2010.

W. Cook and S. Dash. On the matrix-cut rank of polyhedra. Math. Oper. Res., 26:19-30, 2001.

S. Fiorini, S. Massar, S. Pokutta, H. R. Tiwary, and R. de Wolf. Linear vs. semidefinite extended formulations: exponential separation and strong lower bounds. In Proceedings of the 44th symposium on Theory of Computing, pages 95-106. ACM, 2012.

N. Gillis and F. Glineur. On the geometric interpretation of the nonnegative rank. Linear Algebra and its Applications, 437(11):2685-2712, 2012.

M. X. Goemans and D. P. Williamson. A new 3/4-approximation algorithm for max sat. SIAM J. Discrete Math., 7:313-321, 1994.

M. X. Goemans and D. P. Williamson. Improved approximation algorithms for maximum cut and satisfiability problems using semidefinite programming. J. Assoc. Comput. Mach., 42:1115-1145, 1995.

J. Gouveia, P. A. Parrilo, and R. R. Thomas. Lifts of convex sets and cone factorizations. Mathematics of Operations Research, 38(2):248-264, 2013a. 
J. Gouveia, R. Robinson, and R. Thomas. Worst-case results for postivite semidefinite rank. arXiv:1305.4600, 2013b.

V. Guruswami and A. K. Sinop. Lasserre hierarchy, higher eigenvalues, and approximation schemes for graph partitioning and quadratic integer programming with psd objectives. In Foundations of Computer Science (FOCS), 2011 IEEE 52nd Annual Symposium on, pages 482-491. IEEE, 2011.

M. Held and R. Karp. The traveling salesman problem and minimum spanning trees. Oper. Res., $18: 1138-1162,1970$.

B. Kalyanasundaram and G. Schnitger. The probabilistic communication complexity of set intersection. SIAM J. Discrete Math., 5:545-557, 1992.

A. R. Karlin, C. Mathieu, and C. T. Nguyen. Integrality gaps of linear and semi-definite programming relaxations for knapsack. In Integer Programming and Combinatoral Optimization, pages 301314. Springer, 2011.

S. Khot. On the power of unique 2-prover 1-round games. In Proceedings of the thiry-fourth annual ACM symposium on Theory of computing, pages 767-775. ACM, 2002.

S. Khot, G. Kindler, E. Mossel, and R. O'Donnell. Optimal inapproximability results for max-cut and other 2-variable csps? SIAM Journal on Computing, 37(1):319-357, 2007.

E. Kushilevitz and N. Nisan. Communication complexity. Cambridge University Press, 1997.

J. B. Lasserre. An explicit equivalent positive semidefinite program for nonlinear 0-1 programs. SIAM J. Optim., 12:756-769, 2002.

L. Lau, R. Ravi, and M. Singh. Iterative Methods in Combinatorial Optimization. Cambridge Texts in Applied Mathematics. Cambridge, 2011.

M. Laurent. A comparison of the Sherali-Adams, Lovász-Schrijver, and Lasserre relaxations for 0-1 programming. Math. Oper. Res., pages 470-496, 2003.

M. Laurent and M. Deza. Geometry of Cuts and Metrics. Springer-Verlag, Berlin, 1997.

L. Lovász and A. Schrijver. Cones of matrices and set-functions and 0-1 optimization. SIAM J. Optim., 1:166-190, 1991.

E. Mossel, R. O'Donnell, and K. Oleszkiewicz. Noise stability of functions with low influences: invariance and optimality. In Foundations of Computer Science, 2005. FOCS 2005. 46th Annual IEEE Symposium on, pages 21-30. IEEE, 2005.

K. Pashkovich. Extended Formulations for Combinatorial Polytopes. PhD thesis, Magdeburg Universität, 2012.

A. A. Razborov. On the distributional complexity of disjointness. Theoret. Comput. Sci., 106(2): 385-390, 1992.

T. Rothvoß. The matching polytope has exponential extension complexity. In Proc. STOC, 2014.

G. Schoenebeck. Linear level lasserre lower bounds for certain k-csps. In Foundations of Computer Science, 2008. FOCS'08. IEEE 49th Annual IEEE Symposium on, pages 593-602. IEEE, 2008. 
G. Schoenebeck, L. Trevisan, and M. Tulsiani. A linear round lower bound for lovász-schrijver sdp relaxations of vertex cover. In Computational Complexity, 2007. CCC'07. Twenty-Second Annual IEEE Conference on, pages 205-216. IEEE, 2007.

H. D. Sherali and W. P. Adams. A hierarchy of relaxations between the continuous and convex hull representations for zero-one programming problems. SIAM J. Discrete Math., 3:411-430, 1990.

M. Singh and K. Talwar. Improving integrality gaps via Chvátal-Gomory rounding. In Approximation, randomization, and combinatorial optimization, volume 6302 of Lecture Notes in Comput. Sci., pages 366-379. Springer, 2010.

V. V. Vazirani. Approximation algorithms. Springer-Verlag, Berlin, 2001. ISBN 3-540-65367-8.

M. Vyve and L. Wolsey. Approximate extended formulations. Math. Program., 105(2):501-522, 2006.

D. P. Williamson and D. B. Shmoys. The design of approximation algorithms. Cambridge University Press, Cambridge, 2011.

L. Wolsey. Heuristic analysis, linear programming and branch and bound. Math. Programming Stud., 13:121-134, 1980.

M. Yannakakis. Expressing combinatorial optimization problems by linear programs. J. Comput. System Sci., 43(3):441-466, 1991.

G. M. Ziegler. Lectures on Polytopes, volume 152 of Graduate Texts in Mathematics. Springer-Verlag, Berlin, 1995. 